\title{
SEARCHING FOR SCATTERERS: HIGH-CONTRAST IMAGING OF YOUNG STARS HOSTING WIDE-SEPARATION PLANETARY-MASS COMPANIONS
}

\author{
Marta L. Bryan $^{1}$, Brendan P. Bowler ${ }^{2,3,8}$, Heather A. Knutson $^{3}$, Adam L. Kraus ${ }^{2}$, Sasha Hinkley ${ }^{4}$, Dimitri Mawet ${ }^{1}$, \\ ERIC L. Nielsen ${ }^{5,6}$, and Sarah C. Blunt ${ }^{5,7}$ \\ ${ }^{1}$ Cahill Center for Astronomy and Astrophysics, California Institute of Technology, 1200 East California Boulevard, MC 249-17, Pasadena, CA 91125, USA \\ ${ }^{2}$ McDonald Observatory and Department of Astronomy, University of Texas at Austin, Austin, TX 78712, USA \\ ${ }^{3}$ Division of Geological and Planetary Sciences, California Institute of Technology, Pasadena, CA 91125, USA \\ ${ }^{4}$ University of Exeter, Physics Department, Stocker Road, Exeter EX4 4QL, UK \\ ${ }^{5}$ SETI Institute, Carl Sagan Center, 189 Bernardo Avenue, Mountain View, CA 94043, USA \\ ${ }^{6}$ Kavli Institute for Particle Astrophysics and Cosmology, Stanford University, Stanford, CA 94305, USA \\ ${ }^{7}$ Department of Physics, Brown University, Providence, RI 02912, USA \\ Received 2016 February 13; revised 2016 May 30; accepted 2016 June 16; published 2016 August 11
}

\begin{abstract}
We have conducted an angular differential imaging survey with NIRC2 at Keck in search of close-in substellar companions to a sample of seven systems with confirmed planetary-mass companions (PMCs) on wide orbits ( $>50 \mathrm{au}$ ). These wide-separation PMCs pose significant challenges to all three possible formation mechanisms: core accretion plus scattering, disk instability, and turbulent fragmentation. We explore the possibility that these companions formed closer in and were scattered out to their present-day locations by searching for other massive bodies at smaller separations. The typical sensitivity for this survey is $\Delta K \sim 12.5$ at $1^{\prime \prime}$. We identify eight candidate companions, whose masses would reach as low as one Jupiter mass if gravitationally bound. From our multi-epoch astrometry we determine that seven of these are conclusively background objects, while the eighth near DH Tau is ambiguous and requires additional monitoring. We rule out the presence of $>7 M_{\text {Jup }}$ bodies in these systems down to $15-50$ au that could be responsible for scattering. This result combined with the totality of evidence suggests that dynamical scattering is unlikely to have produced this population of PMCs. We detect orbital motion from the companions ROXs 42B b and ROXs 12 b, and from this determine 95\% upper limits on the companions' eccentricities of 0.58 and 0.83 respectively. Finally, we find that the $95 \%$ upper limit on the occurrence rate of additional planets with masses between 5 and $15 M_{\text {Jup }}$ outside of 40 au in systems with PMCs is $54 \%$.
\end{abstract}

Key words: methods: statistical - planetary systems - techniques: high angular resolution

\section{INTRODUCTION}

Observational studies of exoplanet systems present a unique opportunity to probe the mechanisms behind planet formation. Over the past decade, surveys using a variety of techniques (radial velocity, transit, microlensing, direct imaging) have revealed a multitude of new systems with astoundingly diverse properties. Many of these systems are difficult to explain within the framework of standard planet formation theories (e.g., Pollack et al. 1996; Boss 2006), and have forced theorists and observers alike to re-evaluate their narratives for planet formation and migration. Perhaps one of the biggest challenges for planet formation models comes from direct imaging surveys, which have uncovered of a new population of young planetary-mass companions (PMCs) $\quad\left(<15 M_{\text {Jup }}\right)$ located beyond $50 \mathrm{au}$.

Chauvin et al. (2004) discovered a $5 M_{\text {Jup }}$ companion 2 M1207 b orbiting 55 au away from a $25 M_{\text {Jup }}$ brown dwarf. Shortly afterwards, additional discoveries of other wideseparation PMCs such as AB Pic b (Chauvin et al. 2005), DH Tau b (Itoh et al. 2005), and CHXR 73 b (Luhman et al. 2006) drove observers and theorists to question how this growing population of objects formed (Lodato et al. 2005; Boss 2006). To date, fifteen PMCs at large orbital distances have been confirmed, most of which are extremely young, $<10$ Myr old (Bowler et al. 2014). Three possible formation routes have been proposed for these wide-separation planets,

\footnotetext{
${ }^{8}$ McDonald Prize Fellow.
}

including direct collapse from molecular cloud fragmentation, disk instability, and core accretion plus gas capture, but all three have significant problems explaining this population of PMCs.

In the process of molecular cloud fragmentation, all stellar and substellar objects begin as opacity-limited fragments with masses of a few Jupiter masses and subsequently begin to accrete gas from the molecular cloud (Low \& LyndenBell 1976). Hydrodynamical star formation simulations have shown that in order to stop accretion at brown dwarf or planetary masses, PMCs must either form at nearly the same time that the circumstellar envelope is exhausted, or else they must be dynamically ejected from the densest regions of gas before they are able to accrete much additional mass (Bate et al. 2002; Bate 2009, 2012). This mechanism has a very difficult time producing binaries with the high mass ratios needed to match the observed wide-separation planetary systems.

In models of disk instability, gas giant planets form rapidly via fragmentation of a gravitationally unstable disk. For this model to work, the disk needs to be massive enough and cold enough to gravitationally collapse. In the majority of scenarios, the disk surface densities beyond 100 au are too low for gravitational instability to operate. While some models show that disk fragmentation can occur outside 100 au (Boss 2006; Dodson-Robinson et al. 2009; Vorobyov 2013), the fragments rarely survive to become full-fledged planetary embryos. This low survival probability is due to processes such as inward migration and accretion onto the host star, or ejection from the system due to dynamical interactions. While it has been 
Table 1

Target Sample

\begin{tabular}{|c|c|c|c|c|c|c|c|c|c|}
\hline System & $\begin{array}{l}\text { R.A. } \\
\text { (J2000) }\end{array}$ & $\begin{array}{l}\text { decl. } \\
(\text { J2000) }\end{array}$ & Pri. SpT & $\begin{array}{c}m_{K} \\
(\mathrm{mag})\end{array}$ & $\begin{array}{c}m_{R} \\
(\mathrm{mag})\end{array}$ & $\begin{array}{l}\text { Dist. } \\
\text { (pc) }\end{array}$ & $\begin{array}{l}M_{\text {comp }} \\
\left(M_{\text {Jup }}\right)\end{array}$ & $\begin{array}{l}\text { Age } \\
\text { (Myr) }\end{array}$ & References \\
\hline 2M0122-2439 & 012250.94 & -243950.6 & M3.5 & $9.20 \pm 0.03$ & 13.6 & $36 \pm 4$ & $12-25$ & $120 \pm 10$ & $(1),(2),(3)$ \\
\hline DH Tau & 042941.56 & +263258.3 & M1 & $8.18 \pm 0.03$ & 12.1 & $145 \pm 15$ & $12_{-4}^{+10}$ & $1-2$ & $(4),(6),(10),(13)$ \\
\hline $2 \mathrm{M} 1626-2527$ & 162627.75 & -252724.7 & M0 & $9.21 \pm 0.03$ & 15.8 & $120 \pm 10$ & $\cdots$ & $8_{-3}^{+4}$ & $(2),(4),(6)$ \\
\hline ROXs 12 & 162628.10 & -252647.1 & M0 & $9.10 \pm 0.03$ & 13.5 & $120 \pm 10$ & $12-20$ & $8_{-3}^{+4}$ & $(2),(4),(6),(12)$ \\
\hline ROXs 42B & 163115.02 & -243243.7 & M1 & $8.67 \pm 0.02$ & 13.4 & $120 \pm 10$ & $6-14$ & $7_{-2}^{+3}$ & $(2),(4),(5),(6),(8)$ \\
\hline HN Peg & 214431.33 & +14 4619.0 & G0 & $4.56 \pm 0.04$ & 5.6 & $18.4 \pm 0.3$ & $21 \pm 9$ & $300-400$ & $(6),(10),(11)$ \\
\hline
\end{tabular}

References. (1) Bowler et al. (2013), (2) Carlsberg Meridian Catalogue (2011), (3) Cutri et al. (2013b), (4) Kraus et al. (2014), (5) Currie et al. (2014), (6) Cutri et al. (2013a), (7) Reid \& Walkowicz (2006), (8) Zacharias et al. (2012), (9) Metchev \& Hillenbrand (2006), (10) Zacharias et al. (2005), (11) Luhman et al. (2007), (12) Skiff (2013), (13) Itoh et al. (2005).

suggested that disk instability could be effective for exceptionally massive disks, this is an extremely limited region of disk parameter space (Vorobyov 2013).

Finally, in the core accretion model, cores grow via successions of two-body collisions between solids until they are massive enough to start runaway gas accretion (Pollack et al. 1996; Alibert et al. 2005). In situ formation of massive wide-separation planets through core accretion is unlikely since the timescale to grow massive cores at these separations is longer than the observed lifetimes of protoplanetary disks. However, recent simulations of core formation via pebble accretion have shown that gas giant cores can form at separations out to 50 au comfortably before the gas in the disk dissipates (Lambrechts \& Johansen 2012). Furthermore, it might be possible for these giant planets to form closer to the star and be subsequently scattered out beyond 100 au by another planet in the system. One potential scenario is that if multiple planet-planet scatterings occur, these giant planets could permanently end up in stable, wide-separation orbits (Scharf \& Menou 2009). Simulations have shown that in this case, these wide-separation planets have high eccentricities of $>0.5$ (Scharf \& Menou 2009; Nagasawa \& Ida 2011). While planet-planet scattering seems to be a potential solution, it requires another body in the system at least as massive as the wide separation planets.

Thus far, despite hundreds of hours of AO imaging, only one multi-planet system has been confirmed with this technique, HR 8799 (Marois et al. 2008, 2010). Recently, two surveys have found evidence of additional planets in two more systems: LkCa 15 and HD 100546 (Kraus \& Ireland 2012; Currie et al. 2015; Quanz et al. 2015; Sallum et al. 2015). Searching for additional planets in these directly imaged systems is critical to understanding the formation and orbital evolution of planets at wide separations, a parameter space currently explored solely by the direct imaging technique.

In this study, we explore the possibility that the observed wide-separation PMCs formed closer in to their host stars, and were scattered out to their present day locations by another massive companion within the system. We conducted an angular differential imaging (ADI) survey with NIRC2 at Keck in search of close-in substellar companions to a sample of seven systems with confirmed PMCs on extremely wide orbits. Our observations are sensitive to companions at significantly lower masses and smaller separations than previous studies of these systems, and allow us to place much stronger constraints on the presence of inner companions. We also use these same systems to calculate the first estimate of the multiplicity of directly imaged planetary systems.

This paper is structured in the following manner. In Section 2 we describe the selected sample of systems and the methods for obtaining the ADI imaging data. In Section 3 we describe the PCA reduction of the images as well as a new method to simultaneously calculate astrometry and relative photometry of candidate companions. Finally, in Section 4 we discuss our results and their implications for the formation mechanisms of this population of wide-separation PMCs.

\section{OBSERVATIONS}

\subsection{Target Selection}

We selected our targets from the sample of 15 systems with confirmed companions beyond 50 au with mass ranges that are either below or straddle the deuterium burning limit $\left(<15 M_{\text {Jup }}\right)$. These systems are as a whole extremely young, which translates into higher sensitivity to lower mass planets at smaller separations. From this larger sample, we selected targets that were observable from Keck and that had previously been imaged only with short integrations. This would allow our deeper follow-up imaging to achieve unprecedented levels of sensitivity in these systems. Altogether, we targeted seven systems: ROXs 42B, ROXs 12, HN Peg, HD 203030, DH Tau, LP 261-75, and 2MASS J012250-243950. Table 1 summarizes the properties of this sample. In addition, we targeted 2MASS J162627744-2527247, which does not have a previously confirmed wide-separation PMC. This star is a wide separation stellar companion to ROXs 12 , located $40^{\prime \prime}$ away. ${ }^{9}$ Not only do the two stars show identical proper motion, but 2M1626-2527 and ROXs 12 also exhibit WISE excesses, indicating that these objects form a wide binary, are diskbearing, and are young.

\subsection{NIRC2 Imaging}

We used the near-infrared imaging camera NIRC2 at the Keck II $10 \mathrm{~m}$ telescope for all of our observations. Adaptive optics imaging was carried out in natural guide star mode using

\footnotetext{
9 There was some confusion with regards to follow-up observations of ROXs 12. The coordinates for ROXs 12 listed in Simbad and in both the discovery and confirmation papers of the PMC ROXs 12b (Ratzka et al. 2005; Kraus et al. 2014) are for 2M1626-2527, which does not have a confirmed PMC. The correct coordinates for ROXs 12 are listed in Table 1.
} 
Table 2

Keck/NIRC2 Observations of PMC Systems

\begin{tabular}{|c|c|c|c|c|c|c|c|c|}
\hline System & UT Date & Filter & $\begin{array}{l}\text { Coronagraph Diam. } \\
\text { (mas) }\end{array}$ & No. of Exp. & $\begin{array}{l}\text { Tot. Exp. Time } \\
\text { (min) }\end{array}$ & $\begin{array}{c}\text { Rot. } \\
\text { (degree) }\end{array}$ & Airmass $^{\mathrm{a}}$ & $\begin{array}{l}\text { FWHM }^{\mathrm{a}, \mathrm{b}} \\
\quad(\mathrm{mas})\end{array}$ \\
\hline $2 \mathrm{M} 0122-2439$ & 2014 Nov 9 & $K_{S}$ & 600 & 30 & 30 & 11.0 & 1.44 & $46.5 \pm 0.6$ \\
\hline DH Tau & 2014 Dec 7 & $K_{S}$ & 600 & 25 & 25 & 57.6 & 1.07 & $45.9 \pm 1.9$ \\
\hline DH Tau & 2015 Nov 04 & $K_{S}$ & 600 & 25 & 25 & 36.7 & 1.01 & $46.3 \pm 1.2$ \\
\hline 2M1626-2527 & 2014 May 13 & $K_{S}$ & 600 & 28 & 28 & 11.3 & 1.44 & $73.6 \pm 11.6$ \\
\hline 2M1626-2527 & 2015 Jun 23 & $K_{S}$ & 600 & 25 & 25 & 9.5 & 1.48 & $45.8 \pm 1.5$ \\
\hline ROXs 12 & 2011 Jun 23 & $K_{P}$ & 300 & 27 & 13.5 & 5.4 & 1.56 & $47.7 \pm 1.6$ \\
\hline ROXs 12 & 2015 Aug 27 & $K_{S}$ & 600 & 20 & 20 & 6.4 & 1.54 & $53.0 \pm 9.3$ \\
\hline ROXs 42B & 2011 Jun 23 & $K_{P}$ & 300 & 46 & 23 & 13.9 & 1.44 & $45.2 \pm 4.9$ \\
\hline ROXs 42B & 2014 May 13 & $K_{S}$ & 600 & 30 & 30 & 12.3 & 1.41 & $60.1 \pm 10.1$ \\
\hline HD 203030 & 2014 Nov 9 & $K_{S}$ & 600 & 60 & 30 & 12.4 & 1.06 & $43.0 \pm 0.3$ \\
\hline HD 203030 & 2015 Jun 3 & $K_{S}$ & 600 & 80 & 40 & 80.9 & 1.02 & $40.4 \pm 1.6$ \\
\hline HN Peg & 2014 Aug 4 & $K_{S}$ & 600 & 50 & 25 & 102.3 & 1.01 & $47.7 \pm 0.7$ \\
\hline HN Peg & 2015 Jun 2 & $K_{S}$ & 600 & 70 & 35 & 32.1 & 1.04 & $39.6 \pm 1.2$ \\
\hline
\end{tabular}

Notes.

${ }^{a}$ Values averaged over the total duration of the observations for each target, which typically spanned 20-30 minutes.

b The reported uncertainty on each FWHM value is the standard deviation of the PSF over the duration of the observations.

the narrow camera. Due to the realignment of the Keck II AO and NIRC2 system in 2015 April, for epochs taken prior to this date we used a plate scale of $0.009952 \pm 0.000002 \mathrm{arcsec} /$ pixel (Yelda et al. 2010), and for epochs taken after this date we used a plate scale of $0.009971 \pm 0.000004 \mathrm{arcsec} / \mathrm{pixel}$ (Service et al. 2016). The field of view (FOV) of the $1024 \times 1024$ array is 10 ". $2 \times 10^{\prime \prime}$. 2 . For each system we obtained a total integration time of $\sim 30$ minutes in ADI mode with an average of $\sim 30^{\circ}$ of sky rotation. For each image, the star was centered behind a coronagraph, which for nearly all images was the 600 mas diameter coronagraph. This coronagraph is partially transparent with $6.65+/-0.10 \mathrm{mag}$ of attenuation in $K s$ band (Bowler et al. 2015a). Due to high noise levels in the lower left quadrant of the detector, we positioned the occulting spot (already fixed at row 430) at column 616. When possible, we observed these systems as they were transiting to maximize the rotation achieved during the observation, which makes post-processing point-spread function (PSF) subtraction more effective. The inner working angle achieved for these observations is 300 mas, while the outer working angle for complete FOV coverage is $\sim 4^{\prime \prime}$. All observations were taken with the $K s$ filter, which maximizes the Strehl ratio while avoiding the high sky backgrounds encountered in $L$ band. We took second epoch data for the systems where we identified a candidate companion.

\section{ANALYSIS}

\subsection{PCA Image Reduction}

After removing bad pixels and cosmic rays and flat-fielding, we applied the Yelda et al. (2010) distortion correction to raw images taken before 2015 April, and applied the updated Service et al. (2016) distortion solution to later epochs. We then used principle component analysis (PCA) to further reduce these images. PCA is an algorithm that has recently been applied to high contrast imaging for increasing the contrast achievable next to a bright star. In short, PCA is a mathematical technique that relies on the assumption that every image in a stack can be represented as a linear combination of its principle orthogonal components, selecting structures that are present in most of the images. The stellar PSF, composed of a sum of orthogonal components, is subtracted from each image, thereby providing access to faint companions at contrasts below the speckle noise. We used a PCA routine following the method presented in Soummer et al. (2012) which uses the KLIP algorithm.

The optimal number of principle components to use in a reduction is set by the trade-off between speckle noise and selfattenuation of the signal of interest. Too few components might not subtract enough speckle noise near the star, and too many may lead to self-subtraction of the planetary signal, reducing the achievable contrasts. In our analysis, we optimized the number of components used for each individual system empirically. We reduced the data for each system with different numbers of principle components, then compared resulting contrast curves that were calibrated for self-attenuation by injecting fake companions. For each system we adopted the number of principle components that corresponded to the most favorable contrast as the optimal number. These ranged from 5 to 20 principle components for systems in our sample.

We found a total of nine candidate companions at a wide variety of separations in the eight systems that we observed. These candidate companions are shown in Figure 1, and the contrast curves for all systems observed are shown in Figure 2. We determine our contrast curves by calculating the noise level in our images as a function of radial distance using the standard deviation on concentric annuli of width FWHM of the stellar PSF. The noise level at each radius is corrected for selfsubtraction by dividing by the self-attenuation at that radius. This self-attenuation factor is calculated by injection and recovery of sources with known magnitudes at different radii. We present the $5 \sigma$ contrast curves in Figure 2, which are simply our noise levels divided by the self-attenuation factor multiplied by a factor of 5 . We list $5 \sigma$ contrast values for a range of angular separations for each target in Table 3 . We note that these contrasts are often limited by small PA rotation and subsequent ADI self-subtraction.

\subsection{Simultaneous Astrometry and Relative Photometry}

Using second epoch data, we can determine whether or not these candidate companions are co-moving. Typical methods for determining the astrometry and photometry of candidate 

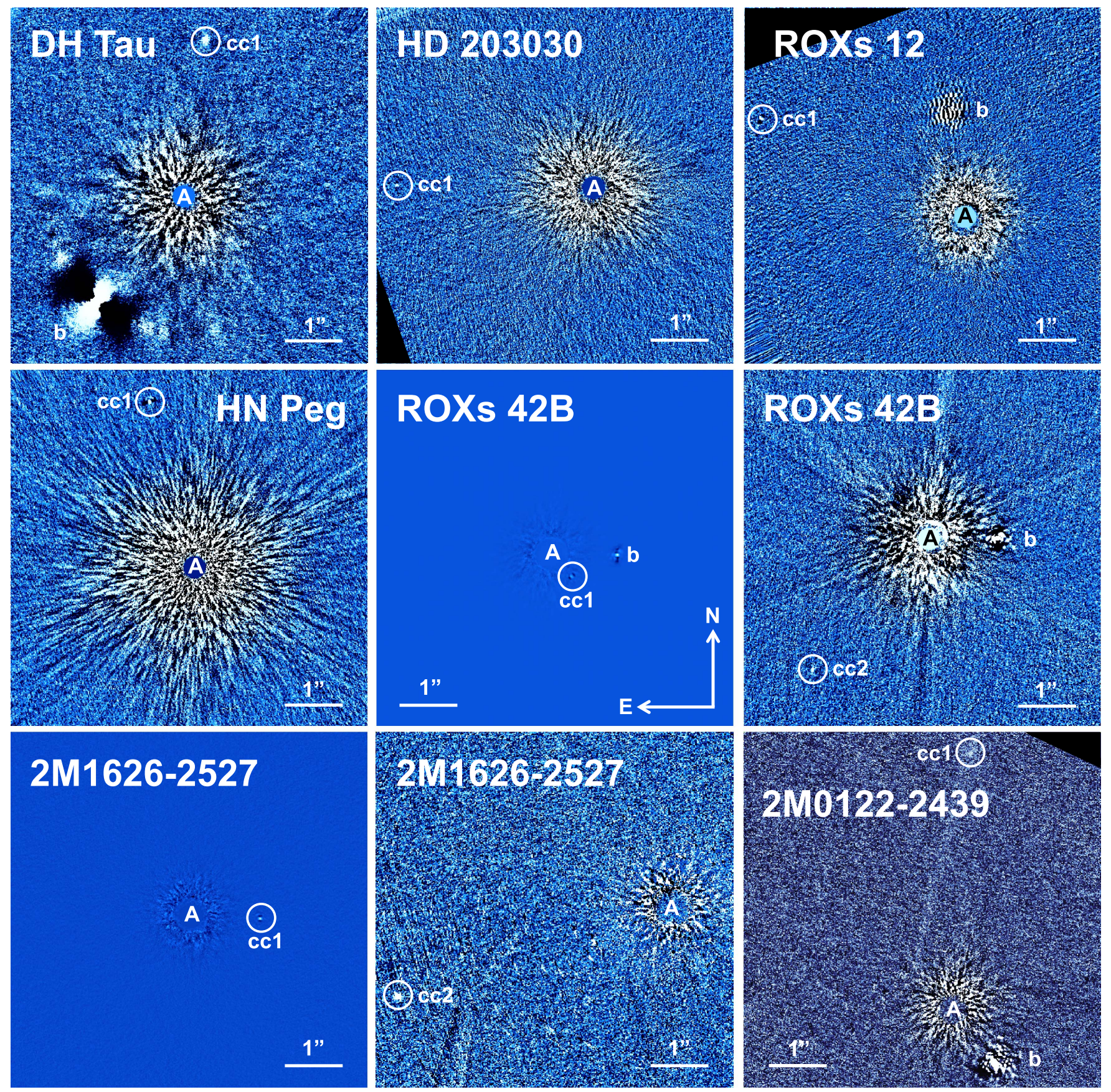

Figure 1. Candidate companions in our sample. All images are north-aligned. ROXs 42B and 2M1626-2527 are shown twice with different stretches to accommodate candidate companions with significantly different flux ratios. Some of the known companions exhibit speckle-like features in these images. This is due to the fact that these bright companions were not masked during the PCA reduction, so some of the PCA components were structured to subtract away the signal of the confirmed companions. The PCA algorithm was able to more successfully subtract away companions with small amounts of rotation (i.e., ROXs 12 b) in comparison to companions with large amounts of rotation (i.e., DH Tau b).

companions use the final post-processed images for these calculations. However, we note that because of self-subtraction, using the final LOCI or PCA images to calculate separations, position angles, and their uncertainties can lead to significant biases in the corresponding photometry and astrometry (e.g., Marois et al. 2010). To avoid this, we developed an MCMC algorithm that simultaneously calculates the astrometry and relative photometry of these candidate companions. For each iteration in the MCMC process, we injected a negative PSF into each individual science image in the vicinity of the companion of interest prior to de-rotation, where we modeled these negative PSFs as Moffat distributions. There were three parameters that we varied with each step during the MCMC routine, namely the negative PSF amplitude, separation, and position angle. We fixed all other free parameters to the values determined from fitting the Moffat distribution to the stellar PSF. The science images with injected negative PSFs were then run through the $\mathrm{PCA}$ reduction routine. The smaller the rms noise at the location of the candidate companion, the better the fit of the negative PSF. The result of this MCMC analysis is a posterior distribution for the amplitude, separation, and position angle of the candidate companions, an example of 
which is shown in Figure 3. Figure 4 compares a reduced image with and without the best-fit negative PSF injected at the best fit separation and position angle of the candidate companion.

In addition to the errors from the candidate companion PDFs for the separation and position angle, after the MCMC program is finished we also account for uncertainties from the registration of the stellar position in each science image, uncertainties from the distortion correction, and uncertainties associated with the plate scale. We also include the +0.252 correction for north alignment to the NIRC2 header position angles (Yelda et al. 2010) for epochs taken before 2015 April, and for subsequent epochs include the +0.262 correction for

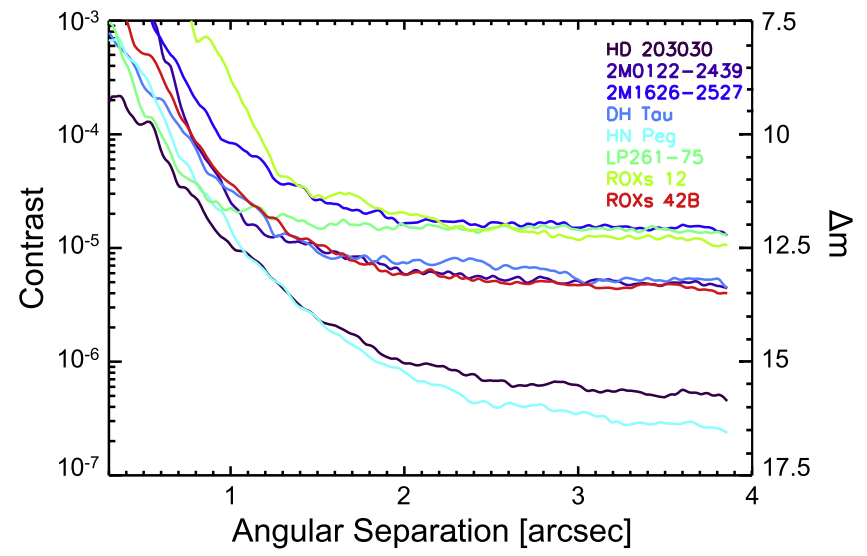

Figure 2. $5 \sigma$ contrast curves for systems in our sample. The stellar flux was modified by a throughput correction (Bowler et al. 2015a) due to attenuation from the coronagraph spot. The curves have been corrected for self-attenuation of the target of interest using a robust injection and recovery technique. Note that the flattening of the contrast curves indicates a background-limited regime, as opposed to speckle limitations. north alignment (Service et al. 2016). This MCMC technique calculates robust uncertainties from the posterior distributions, without the systematic uncertainties that occur when the reduced LOCI or PCA images are used. The best-fit separations and position angles for each candidate companion, as well as confirmed companions in these systems, are presented in Table 4.

While the separation, position angle, and amplitude are the three parameters that were actively varied with each step in the MCMC program, we also track the total flux of the candidate companion. For each link in the chain, we place an aperture at the separation and position angle of that step and sum the number of counts in the aperture. While the size of the aperture for a given companion remains fixed, the aperture size ranged from 4 to 6 pixels depending on the candidate companion FWHM. The aperture moves with the changes in position angle and separation as the MCMC program progresses, producing a posterior distribution of counts for the candidate companion. We note that since we calculate the astrometry and relative photometry simultaneously using this MCMC program, we use the same aperture sizes for both of these steps for a given candidate companion.

We next calculate the contrast relative to the host star, $\Delta m$, for each candidate companion. To determine the flux from the star, the throughput of the occulting spot $(0.0022 \pm 0.0002$, Bowler et al. 2015a), and the sky noise need to be accounted for. The measured counts are a combination of the flux from the star plus the flux from the sky, both attenuated by the throughput of the mask. We denote this combined and attenuated star plus sky flux as $F_{b, \star}$. The corrected flux for the star is shown in Equation (1). $T$ is the throughput (0.0022) of the mask in $K_{S}, F_{\text {sky }}$ is the sky flux, and $F_{\star}$ is the flux from

Table 3

Contrast Curves

\begin{tabular}{|c|c|c|c|c|c|c|c|}
\hline System & $0 . " 5$ & $1^{\prime \prime}$ & $1 " 15$ & $2^{\prime \prime}$ & $2 ! 5$ & $3^{\prime \prime}$ & $3 " .5$ \\
\hline $2 \mathrm{M} 0122-2439$ & $1.4 \times 10^{-3}$ & $2.5 \times 10^{-5}$ & $1.0 \times 10^{-5}$ & $5.9 \times 10^{-6}$ & $5.6 \times 10^{-6}$ & $5.1 \times 10^{-6}$ & $4.8 \times 10^{-6}$ \\
\hline DH Tau & $2.7 \times 10^{-4}$ & $3.2 \times 10^{-5}$ & $9.1 \times 10^{-6}$ & $7.4 \times 10^{-6}$ & $6.9 \times 10^{-6}$ & $5.2 \times 10^{-6}$ & $5.1 \times 10^{-6}$ \\
\hline LP261-75 & $1.6 \times 10^{-4}$ & $2.2 \times 10^{-5}$ & $1.5 \times 10^{-5}$ & $1.6 \times 10^{-5}$ & $1.5 \times 10^{-5}$ & $1.4 \times 10^{-5}$ & $1.5 \times 10^{-5}$ \\
\hline ROXs 12 & $2.7 \times 10^{-2}$ & $3.0 \times 10^{-4}$ & $2.9 \times 10^{-5}$ & $2.0 \times 10^{-5}$ & $1.5 \times 10^{-5}$ & $1.2 \times 10^{-5}$ & $1.3 \times 10^{-5}$ \\
\hline ROXs 42B & $5.1 \times 10^{-4}$ & $3.6 \times 10^{-5}$ & $1.1 \times 10^{-5}$ & $5.8 \times 10^{-6}$ & $5.1 \times 10^{-6}$ & $4.7 \times 10^{-6}$ & $4.8 \times 10^{-6}$ \\
\hline HD 203030 & $1.2 \times 10^{-4}$ & $1.0 \times 10^{-5}$ & $2.4 \times 10^{-6}$ & $9.7 \times 10^{-7}$ & $6.8 \times 10^{-7}$ & $6.2 \times 10^{-7}$ & $4.9 \times 10^{-7}$ \\
\hline
\end{tabular}

Note. All of these contrasts correspond to images taken using the $K_{S}$ filter.
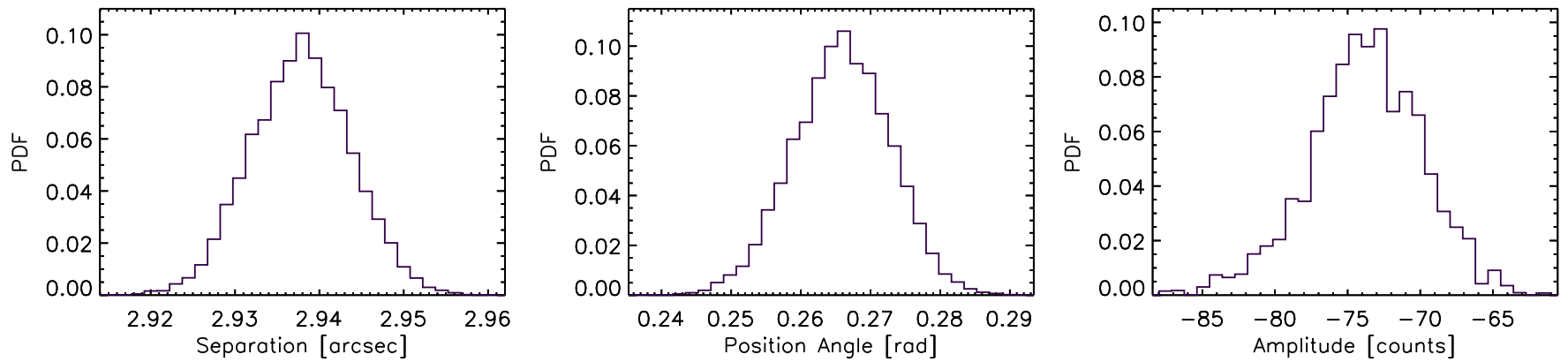

Figure 3. Example posterior PDFs from the MCMC astrometry calculation for the candidate companion in our observations of HN Peg. Note that the amplitude PDF shows the amplitude of the negative PSF injected into the science images. 


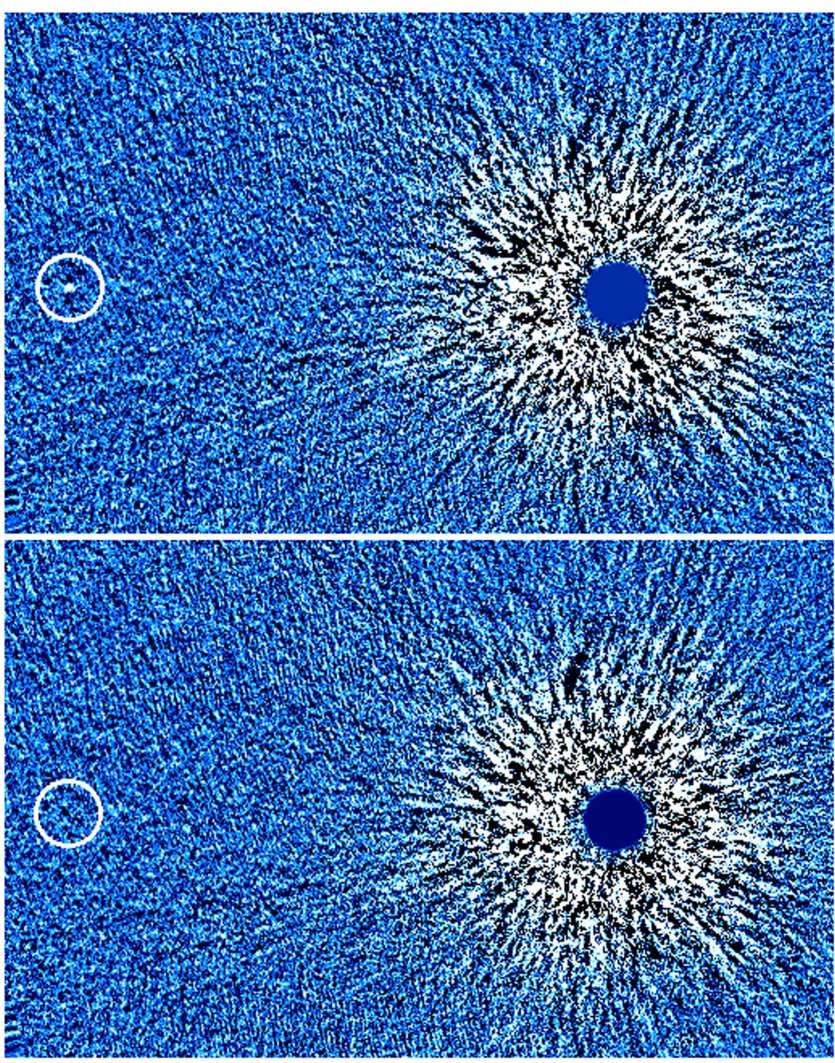

Figure 4. Comparison of PCA images of the HD 203030 candidate companion without (top) and with (bottom) the best fit negative PSF injected. the star corrected for both throughput losses and sky noise.

$$
F_{\star}=\frac{F_{\star, b}}{T}-F_{\text {sky }} .
$$

This calculation is performed for each image in the stack; for a stack of $N$ images, there are $N$ values of $F_{\star}$. The error on this flux value is:

$$
\sigma_{F, \star}=\sqrt{\left(\frac{F_{b, \star}}{T}\right)^{2} \times\left[\left(\frac{\sigma_{\star, b}}{F_{\star, b}}\right)^{2}+\left(\frac{\sigma_{T}}{T}\right)^{2}\right]+\sigma_{\text {sky }}^{2}} .
$$

Here, $\sigma_{\star, b}$ is the standard deviation of the $F_{\star, b}$ values for each image in the stack, $\sigma_{T}$ is the measured error on the throughput, and $\sigma_{\text {sky }}$ is the standard deviation of the sky values calculated for each image.

We obtain the companion flux and its uncertainty from the posterior distribution generated from the MCMC analysis, and subtract off sky noise. The flux ratio between the star and the companion is simply $F_{\text {ratio }}=\frac{F_{\star}}{F_{\text {comp }}}$, and the error on this flux ratio can be propagated analytically:

$$
\sigma_{f r}=F_{\text {ratio }} \times \sqrt{\left(\frac{\sigma_{F, \star}}{F_{\star}}\right)^{2}+\left(\frac{\sigma_{\text {comp }}}{F_{\text {comp }}}\right)^{2}} .
$$

The contrast ratio $\Delta m$ in magnitudes is:

$$
\Delta m=-2.5 \log _{10} F_{\text {ratio }}
$$

\begin{tabular}{|c|c|c|c|c|c|c|}
\hline System & Epoch & Filter & $\rho$ (mas) & P.A. (degree) & $\Delta m$ (mag) & Sep. (au) \\
\hline $2 \mathrm{M} 0122-2439 \mathrm{~b}$ & 2014.8575 & $K_{S}$ & $1450_{-1}^{+1}$ & $216.48_{-0.02}^{+0.02}$ & $4.79 \pm 0.11$ & 52 \\
\hline 2M0122-2439 cc1 & 2014.8575 & $K_{S}$ & $5238_{-16}^{+12}$ & $355.75_{-0.14}^{+0.14}$ & $9.60 \pm 0.20$ & 188 \\
\hline DH Tau cc1 & 2014.9342 & $K_{S}$ & $2726_{-7}^{+7}$ & $351.82_{-0.16}^{+0.16}$ & $8.66 \pm 0.25$ & 395 \\
\hline DH Tau cc1 & 2015.8438 & $K_{S}$ & $2734_{-4}^{+4}$ & $351.35_{-0.10}^{+0.10}$ & $8.45 \pm 0.23$ & 396 \\
\hline DH Tau b & 2014.9342 & $K_{S}$ & $2343_{-1}^{+1}$ & $140.25_{-0.02}^{+0.02}$ & $5.91 \pm 0.20$ & 340 \\
\hline DH Tau b & 2015.8438 & $K_{S}$ & $2339_{-1}^{+1}$ & $139.94_{-0.02}^{+0.02}$ & $5.72 \pm 0.28$ & 340 \\
\hline 2M1626-2527 cc1 & 2014.3644 & $K_{S}$ & $1193_{-14}^{+11}$ & $266.88_{-0.21}^{+0.26}$ & $7.36 \pm 0.23$ & 143 \\
\hline $2 \mathrm{M} 1626-2527 \mathrm{cc} 1$ & 2015.4767 & $K_{S}$ & $1189_{-1}^{+2}$ & $267.93_{-0.02}^{+0.03}$ & $6.93 \pm 0.13$ & 143 \\
\hline 2M1626-2527 cc2 & 2014.3644 & $K_{S}$ & $4883_{-7}^{+8}$ & $107.99_{-0.06}^{+0.06}$ & $7.62 \pm 0.25$ & 586 \\
\hline 2M1626-2527 cc2 & 2015.4767 & $K_{S}$ & $4901_{-3}^{+4}$ & $107.82_{-0.03}^{+0.03}$ & $7.11 \pm 0.13$ & 589 \\
\hline ROXs $12 \mathrm{cc} 1$ & 2011.4767 & $K_{P}$ & $3811_{-10}^{+10}$ & $65.29_{-0.07}^{+0.08}$ & $\ldots$ & 457 \\
\hline ROXs 12 cc 1 & 2015.6548 & $K_{S}$ & $3877_{-14}^{+15}$ & $64.12_{-0.09}^{+0.09}$ & $8.51 \pm 0.13$ & 465 \\
\hline ROXs $12 \mathrm{~b}$ & 2011.4767 & $K_{P}$ & $1778_{-1}^{+1}$ & $8.90_{-0.08}^{+0.08}$ & $\ldots$ & 213 \\
\hline ROXs $12 b$ & 2015.6548 & $K_{S}$ & $1786_{-1}^{+1}$ & $8.18_{-0.30}^{+0.29}$ & $4.30 \pm 0.13$ & 214 \\
\hline ROXs 42B cc1 & 2011.4767 & $K_{P}$ & $580_{-1}^{+1}$ & $224.06_{-0.06}^{+0.06}$ & $\ldots$ & 70 \\
\hline ROXs 42B cc1 & 2014.3644 & $K_{S}$ & $525_{-1}^{+1}$ & $227.27_{-0.06}^{+0.06}$ & $6.23 \pm 0.27$ & 63 \\
\hline ROXs $42 \mathrm{~B} \mathrm{cc} 2$ & 2011.4767 & $K_{P}$ & $3037_{-11}^{+14}$ & $138.99_{-0.15}^{+0.14}$ & $\ldots$ & 365 \\
\hline ROXs 42B cc 2 & 2014.3644 & $K_{S}$ & $2991_{-7}^{+8}$ & $138.27_{-0.12}^{+0.10}$ & $8.37 \pm 0.25$ & 359 \\
\hline ROXs 42B b & 2011.4767 & $K_{P}$ & $1173_{-1}^{+1}$ & $270.06_{-0.01}^{+0.01}$ & $\ldots$ & 141 \\
\hline ROXs $42 \mathrm{~B} \mathrm{~b}$ & 2014.3644 & $K_{S}$ & $1170_{-1}^{+1}$ & $270.55_{-0.01}^{+0.01}$ & $6.16 \pm 0.35$ & 140 \\
\hline HD $203030 \mathrm{cc} 1$ & 2014.8575 & $K_{S}$ & $3379_{-16}^{+12}$ & $89.33_{-0.14}^{+0.14}$ & $11.17 \pm 0.15$ & 139 \\
\hline HD $203030 \mathrm{cc} 1$ & 2015.4219 & $K_{S}$ & $3263_{-7}^{+10}$ & $89.76_{-0.22}^{+0.16}$ & $11.46 \pm 0.20$ & 134 \\
\hline HN Peg cc1 & 2014.5918 & $K_{S}$ & $2931_{-5}^{+4}$ & $19.06_{-0.11}^{+0.13}$ & $12.63 \pm 0.6$ & 54 \\
\hline HN Peg cc1 & 2015.4192 & $K_{S}$ & $2933_{-2}^{+2}$ & $15.22_{-0.05}^{+0.05}$ & $12.13 \pm 0.48$ & 54 \\
\hline
\end{tabular}

Table 4

Candidate PMC Astrometry and Photometry

Note. We do not list $\Delta m$ for the 2011 epochs because we did not have throughput measurements for the 300 mas coronagraph in $K_{P}$. Uncertainties in the astrometry and distance estimates to these systems typically lead to errors in separation of 5-40 au. 

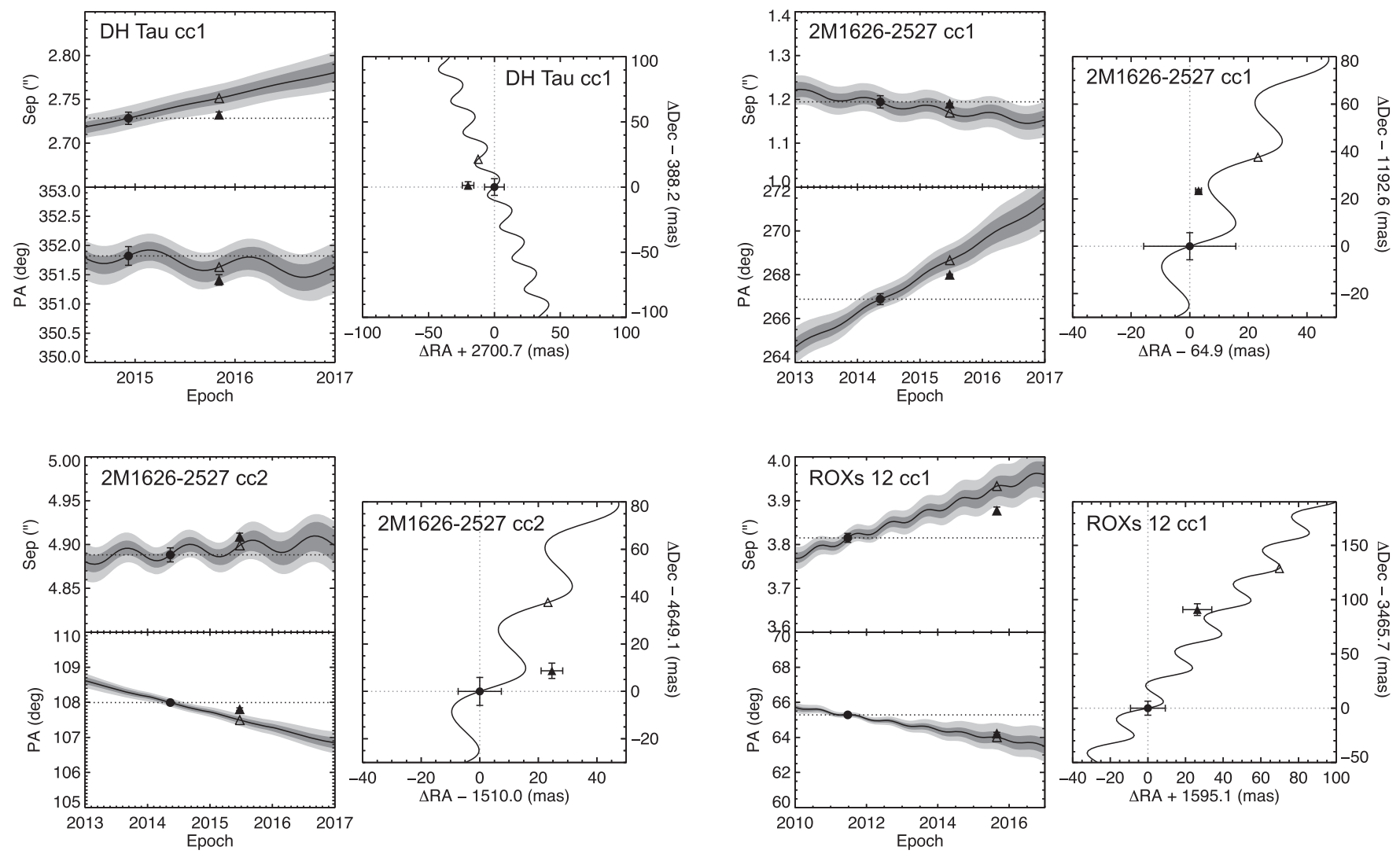

Figure 5. These plots show how the candidate companion's astrometry compares to expected trajectories of a co-moving object and a stationary background object. The first epoch of astrometry is denoted by a filled circle, and the second epoch is denoted by a filled triangle. The open triangles denote the expected astrometry of a stationary background object at the second epoch. The dark and light gray regions represent the one and two sigma errors on the predicted background tracks, respectively. These errors include uncertainties in the distance to the system, proper motion, and astrometry from the reference epoch. If the candidate companion was bound to the star, the second epoch triangles would fall on the horizontal dotted line (separation and position angle would not change as a function of time, except due to orbital motion). Top left: DH Tau candidate companion (cc) 1. Top right: 2M1626-2527 cc1. Bottom left: 2M1626-2527 cc2. Bottom right: ROXs 12 cc1.

Finally, the error on $\Delta m$ is given by:

$$
\sigma_{\Delta m}=\frac{2.5}{\ln 10} \times \frac{\sigma_{f r}}{F_{\text {ratio }}} .
$$

We present the $\Delta m$ values for each candidate and confirmed companion in Table 4 . We note that because 2M0122-2439 $\mathrm{cc} 1$ is so faint we were unable to use our MCMC analysis to calculate the astrometry of that companion (the MCMC chains failed to converge). Instead, we used centroiding on the final image to obtain the separation and position angle of the candidate companion, and adopted the robust errors calculated for the faint HD $203030 \mathrm{cc} 1$. Furthermore, since the candidate companion near 2M0122-2439 appears to be extended (its FWHM is about twice that of the stellar PSF), we conclude that is likely a background galaxy, not a bound planet, and exclude it from the rest of the analysis.

Our astrometry conclusively shows that seven of the remaining eight candidate companions are background objects, while the nature of the candidate companion near DH Tau is ambiguous. Figures 5 and 6 show the relative astrometry of each candidate companion compared to the expected background track of a stationary object. The candidate companion background track plots clearly show that the second epoch astrometry falls on or near the predicted track for a stationary background object. While some of the second epoch astrometry measurements do not fall precisely on the expected track of a stationary background object, we note that this could be due to small errors in proper motion or distance, which would affect the predicted trajectory of a distant stationary object. We note that ROXs 42B cc1 was previously identified as likely a background object in the literature (Currie et al. 2014; Kraus et al. 2014) but our astrometry conclusively shows that it is a background object. For DH Tau cc1, while the second epoch astrometry falls close to the co-moving line, uncertainties on the expected trajectory of a background object make comovement ambiguous. The separation of a stationary object at the second epoch differs by $\sim 2.9 \sigma$ from the separation we find for DH Tau cc1. Zhou et al. (2014) published Hubble Space Telescope UVIS optical photometry for the DH Tau system but they did not report a detection of our candidate companion. They presented detection limits in both $i$ and $z$ filters, which we can use to place limits on the colors of DH Tau cc1. We find that the bluest DH Tau cc1 could be is $i-K=7.8 \mathrm{mag}$. Furthermore, line of sight visual extinction is low, $0.0-1.5 \mathrm{mag}$ (Strom et al. 1989; White \& Ghez 2001). This apparently red color further motivates additional follow-up for the potentially bound DH Tau cc1. A third epoch taken when DH Tau is next observable end of 2016 would conclusively determine whether or not DH Tau cc1 is a bound object.

We also plot the relative astrometry for the previously confirmed companions to ROXs 12, ROXs 42B, and 2M01222439 in Figure 7. We have included astrometry from the literature in addition to the data presented in this paper. These 

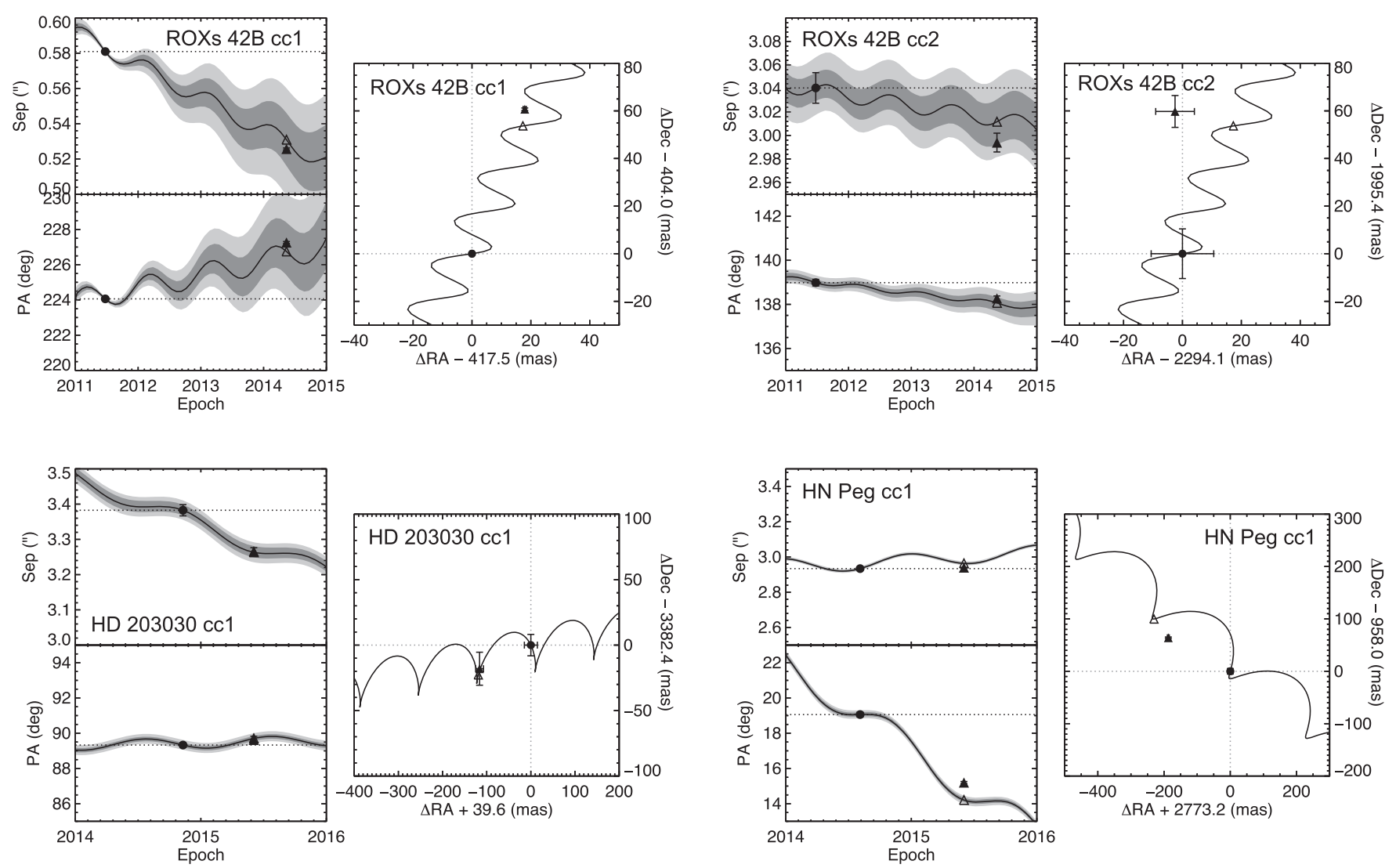

Figure 6. Background track plots for four of our candidate companions. See Figure 5 for more details. Top left: ROXs 42B cc1. Top right: ROXs 42B cc2. Bottom left: HD $203030 \mathrm{cc} 1$. Bottom right: HN Peg cc1.

plots show that follow-up astrometry generally fall near the dotted line denoting co-moving objects. We do not plot the relative astrometry for $\mathrm{DH}$ Tau $\mathrm{b}$, since there are significant systematic offsets for measurements of the companion position angle and separation among previous epochs spanning 1999 through 2013. Table 5 lists literature astrometry measurements that we used for the confirmed companions in each of these three systems.

\subsection{Orbital Motion}

We tested ROXs 42B b, 2M0122-2439 b, and ROXs 12 b for evidence of orbital motion. Assuming a face on, circular orbit, we find that between the first and last epoch of ROXs 42B b, the maximum amount of change we would expect to see in position angle is $0^{\circ} .6$. The actual change in PA between the first and last epochs is $0.49 \pm 00^{\circ} 02$. We performed a linear fit to all epochs with uncertainties in PA and in separation and compared these to the best-fit constants using evidence ratios. Evidence ratios use Akaike's information criterion (AIC) to quantitatively compare models. They are equal to the ratio of each model's Akaike weights, which are a measure of the strength of evidence for a model. An evidence ratio of 9 comparing model 1 to model 2 would mean that model 1 is nine times more likely than model 2 given the data. We label linear fits as preferred if the slope of the line differs from zero by $2-4 \sigma$, and highly preferred if this slope is $>4 \sigma$ away.

In PA we find the evidence ratio comparing a linear to constant fit for ROXs 42B b to be $>10^{4}$, and in separation the evidence ratio is 62 . The best fit slope of the linear fit in PA is $0.1703 \pm 0.0049{\mathrm{deg} \mathrm{yr}^{-1}}$, and in separation is $-0.00132 \pm$
$0.00029 \operatorname{arcsec} \mathrm{yr}^{-1}$. We therefore conclude that the linear fits are highly preferred, suggesting that the displacements that we see in $\mathrm{PA}$ and separation over time are due to the orbital motion of ROXS 42B b.

For the confirmed companion orbiting 2M0122-2439, at a separation of only 52 au we would expect this companion to have moved by 1.3 in PA between the first and last epochs assuming a circular, face-on orbit. However, we only find a change in PA of $0.2 \pm 0^{\circ} .2$ between the first and last epochs. Given that the change in PA is consistent with zero, and the evidence ratio for the separation of the companion favors a constant over a linear fit, we conclude that we do not find evidence of orbital motion for 2M0122-2439 b.

Finally, we assess whether orbital motion is evident for the confirmed companion ROXs $12 \mathrm{~b}$. Between the first and last epochs, assuming a face-on circular orbit we would expect to see a change in PA of $1^{\circ} .6$. We find a change of $2^{\circ} .1 \pm 0.9$. Evidence ratios comparing best linear fits to best fit constants through all four epochs including uncertainties are $4.5 \times 10^{3}$ in PA and $10^{4}$ in separation. The best fit slope of the linear fit in

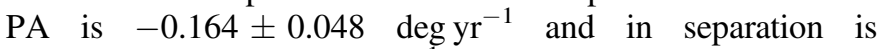
$0.00058 \pm 0.00032 \operatorname{arcsec}_{\mathrm{yr}}^{-1}$. We conclude that linear fits are preferred, and that we likely see orbital motion from ROXs $12 \mathrm{~b}$.

Using multiple epochs of astrometry allows us to constrain the orbits of ROXs 42B b and ROXs 12 b. To fit each orbit we use an updated implementation of the Rejection Sampling Monte Carlo method described in De Rosa et al. (2015), based on the method of Ghez et al. (2008). This technique generates an initial orbit with semi-major axis $(a)$ of unity and position 

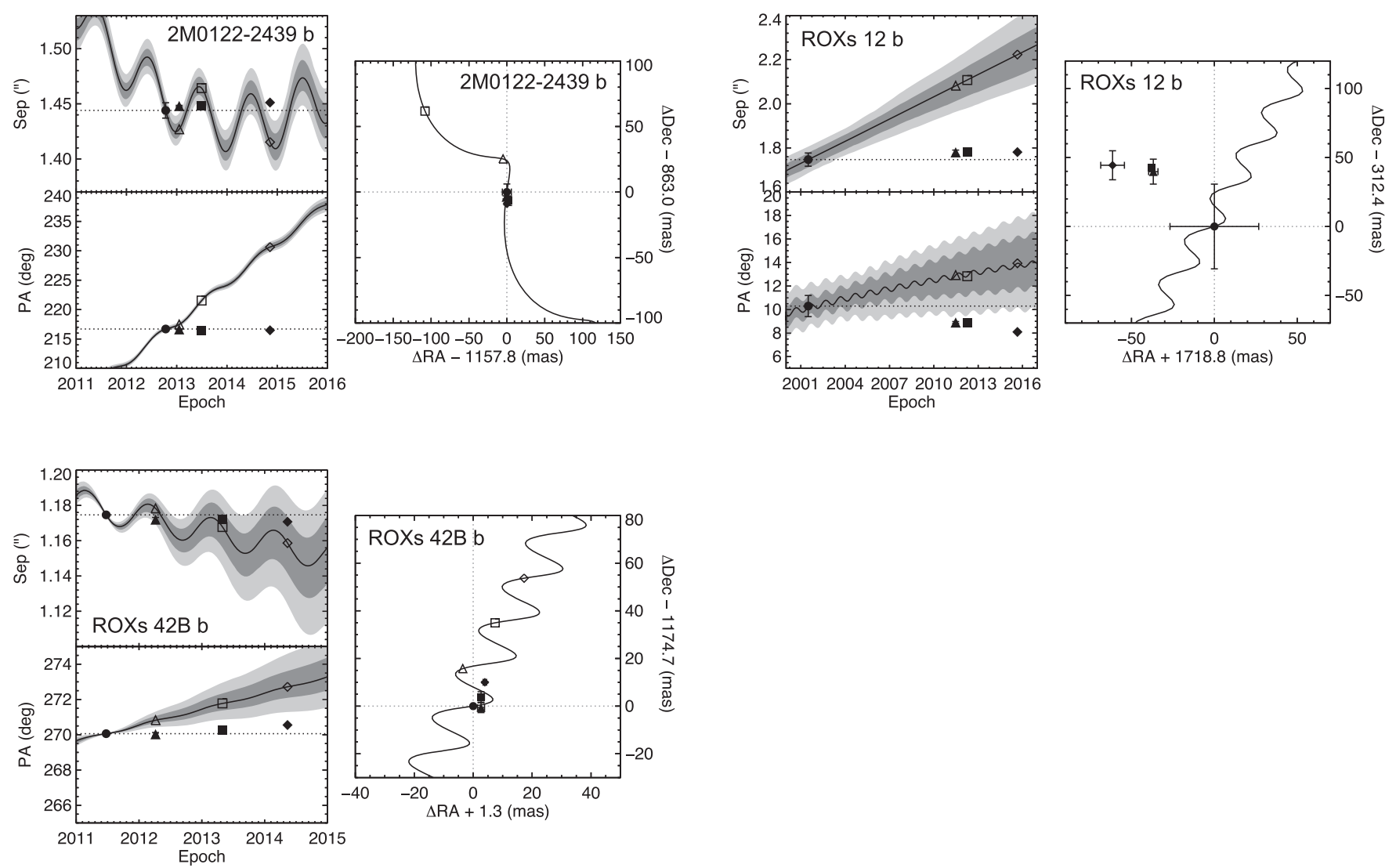

Figure 7. These background track plots show the astrometry of three previously confirmed companions in our sample. Top left: $2 \mathrm{M} 0122-2439$ b. Top right: ROXs 12 b. Bottom left: ROXs 42B b. In the 2M0122-2439 plot, we include two additional epochs of data in 2012 and 2013 from Bowler et al. (2013). In the ROXs 12 plot, we include two additional epochs, in 2001 from Ratzka et al. (2005), and in 2012 from Kraus et al. (2014). In the ROXs 42B plot, we include two additional epochs, in 2001 from Ratzka et al. (2005), and in 2012 from Kraus et al. (2014). The plots for ROXs 12 b and ROXs 42B b show evidence of orbital motion. See Section 3.3 for details.

Table 5

Literature Measurements of Confirmed PMC Astrometry

\begin{tabular}{ccccc}
\hline \hline Companion & Epoch & $\rho$ (mas) & P.A. (degree) & References \\
\hline $\begin{array}{c}\text { 2M0122- } \\
\text { 2439 b }\end{array}$ & 2012.7808 & $1444 \pm 7$ & $216.7 \pm 0.2$ & $(3)$ \\
2M0122- & 2013.0493 & $1448.6 \pm 0.6$ & $216.4 \pm 0.08$ & $(3)$ \\
2439 b & & & & \\
2M0122- & 2013.4959 & $1448 \pm 4$ & $216.47 \pm 0.07$ & $(3)$ \\
2439 b & & & & \\
2M0122- & 2013.6258 & $1488 \pm 3$ & $216.52 \pm 0.09$ & $(5)$ \\
2439 b & & & & \\
ROXs 12 b & 2001.5014 & $1747 \pm 30$ & $10.3 \pm 0.9$ & $(2)$ \\
ROXs 12 b & 2012.2575 & $1783.0 \pm 1.8$ & $8.85 \pm 0.06$ & $(1)$ \\
ROXs 42B b & 2001.5014 & $1137 \pm 30$ & $268.0 \pm 1.5$ & $(2)$ \\
ROXs 42B b & 2005.2904 & $1157 \pm 10$ & $268.8 \pm 0.6$ & $(4)$ \\
ROXs 42B b & 2008.5479 & $1160 \pm 10$ & $269.7 \pm 1.0$ & $(4)$ \\
ROXs 42B b & 2012.2575 & $1172.0 \pm 1.2$ & $270.03 \pm 0.10$ & $(1)$ \\
ROXs 42B b & 2013.3233 & $1172.5 \pm 1.2$ & $270.25 \pm 0.10$ & $(1)$ \\
\hline
\end{tabular}

References. (1) Kraus et al. (2014), (2) Ratzka et al. (2005), (3) Bowler et al. (2013), (4) Currie et al. (2014), (5) Bowler et al. (2015b).

angle of nodes $(\Omega)$ of 0 , with eccentricity $(e)$, inclination angle $(i)$, argument of periastron $(\omega)$, and epoch of periastron passage $\left(T_{0}\right)$ drawn from the appropriate probability distribution: uniform for $e, \omega, T_{0}$, and uniform in $\cos (i)$, and we use Kepler's third law to generate the period from a fixed system mass. We then scale $a$ and rotate $\Omega$ to fit a single observational epoch, with observational errors included by adding Gaussian random noise to the observed separation and position angle for that epoch with $\sigma$ equal to the observational errors. Stellar mass and distance for each trial are both drawn from Gaussian distributions with medians at the measurements and standard deviations of the measurement uncertainties. Unlike De Rosa et al. (2015) where all potential orbits were shifted and scaled to the earliest epoch, here we randomly select an epoch for each orbit, which avoids the fit being biased toward the first epoch.

The algorithm has also been modified at the rejection sampling step: previously we proceeded one epoch at a time, rejecting ill-fitting orbits at each epoch. In this version the chisquare for the newly scaled orbit is calculated for all the remaining epochs, and then the orbit is accepted if a uniform random variable is less than $e^{-\frac{\chi^{2}}{2}}$ and rejected otherwise. Mathematically this is the same operation as we used previously, but it allows for higher computational efficiency in the face of outliers, since the rejection test can be scaled to the minimum value of $\chi^{2}$ reached for the given astrometry, with orbits now accepted if the random variable is less than $e^{-\frac{x^{2}}{2}} / e^{-\frac{x_{\min }^{2}}{2}}$. This method will be described in more detail in S. C. Blunt et al. (2016, in preparation).

This rejection sampling technique produces identical posterior probability distributions to those generated by $\mathrm{MCMC}$, but requires much less computational time for astrometry covering short arcs of an orbit, as demonstrated in De Rosa et al. (2015) 

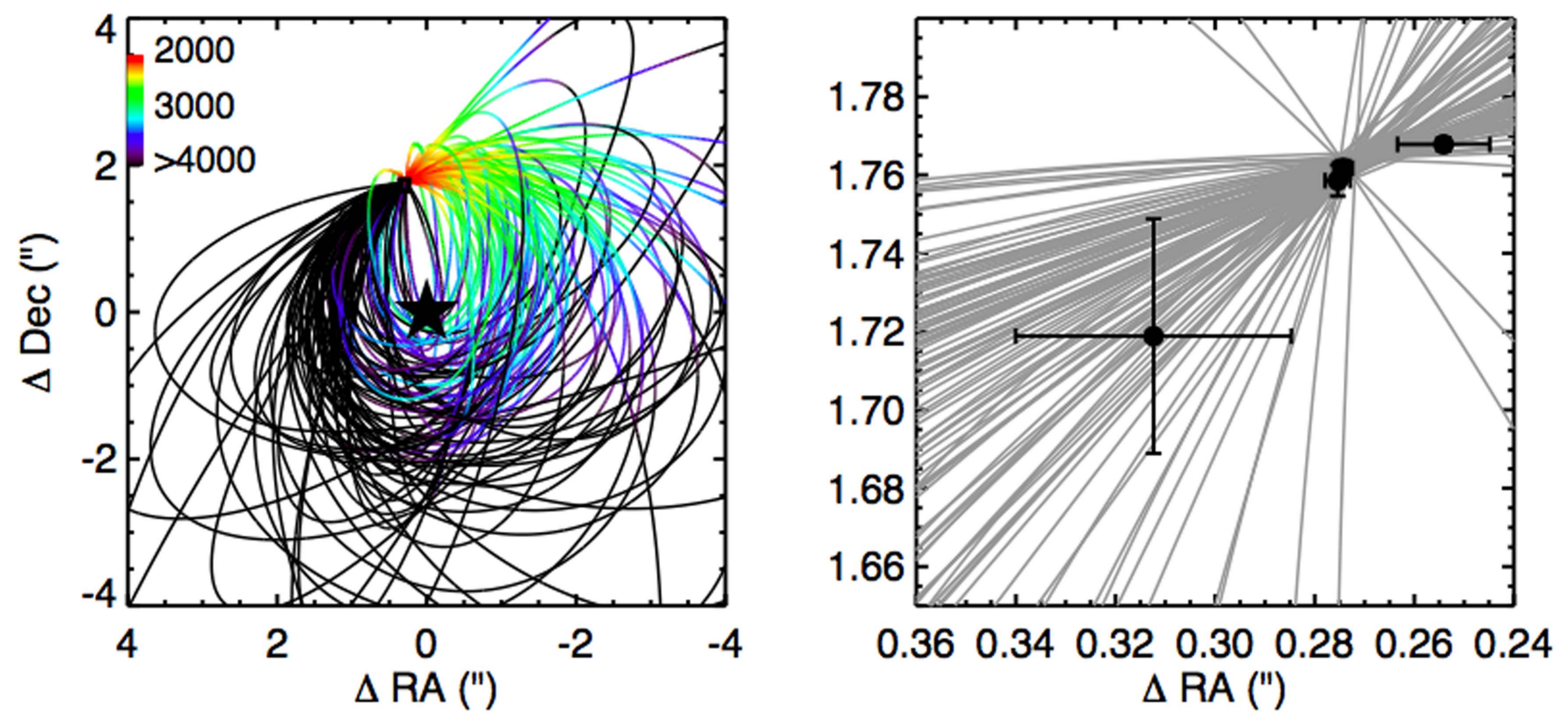

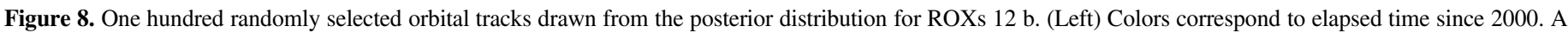

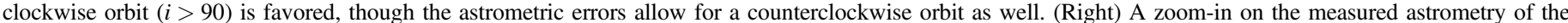
system and the same 100 orbital tracks. Future high-precision astrometric monitoring of the system should improve the constraints on allowable orbits.
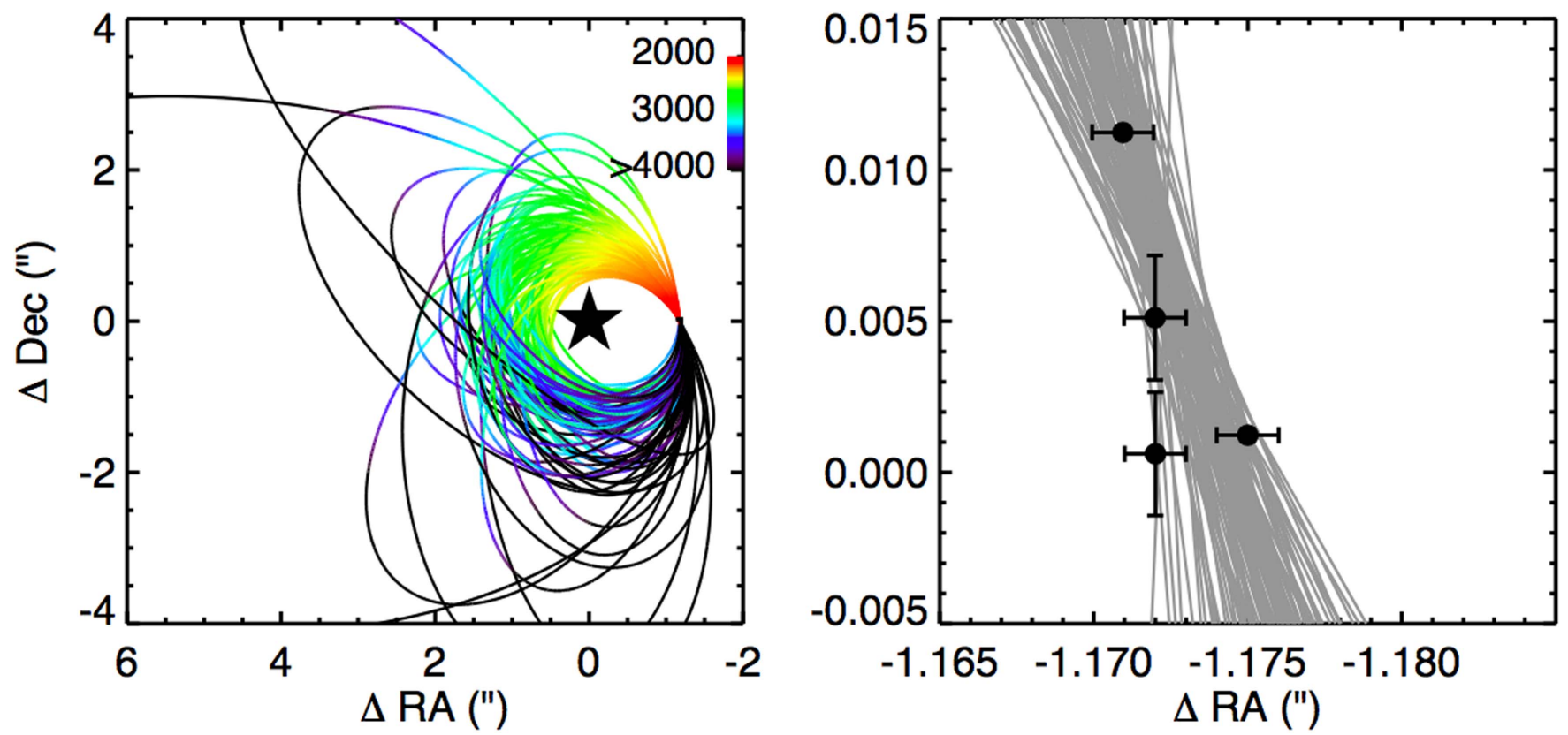

Figure 9. Orbital tracks for ROXs 42B b. See Figure 8 for details. Generally a face on $(i \lesssim 50)$, circular $(e \lesssim 0.5)$ orbit is preferred.

for the exoplanet 51 Eri b. In the very long-period orbits presented here we find that even after $10^{10}$ steps of Metropolis Hastings MCMC the chains have not converged, though the posteriors are broadly similar to those generated by the Rejection Sampling method.

Figures 8 and 9 show the range of Keplerian orbits consistent with the available astrometry for ROXs $12 \mathrm{~b}$ and ROXs $42 \mathrm{~B} \mathrm{~b}$ respectively, while Figures 10 and 11 show the posterior distributions for the orbital parameters that were fit for ROXs $12 \mathrm{~b}$ and ROXs 42B b, respectively. We note that even with a small fraction of orbital coverage, fitting orbits and obtaining marginal constraints on the corresponding parameters is useful. For example, several recent studies have fit the small orbital coverage observed for Fomalhaut $b$, and find that they can constrain the eccentricity of this object to high values (Kalas et al. 2013; Beust et al. 2016). Furthermore, detected orbital motion of the low mass brown dwarfs PZ Tel b and GQ Lup b appears to constrain their eccentricities to high values (Ginski et al. 2014).

While the eccentricities of these PMCs are poorly constrained, we do find that low to moderate eccentricities are favored. The $95 \%$ upper limits on the eccentricities of ROXs $42 \mathrm{~B}$ b and ROXs $12 \mathrm{~b}$ are 0.58 and 0.83 respectively. Previous studies have run scattering simulations to test if these wideseparation (>100 au) PMCs can form via planet-planet scattering. These simulations showed that for giant planets 


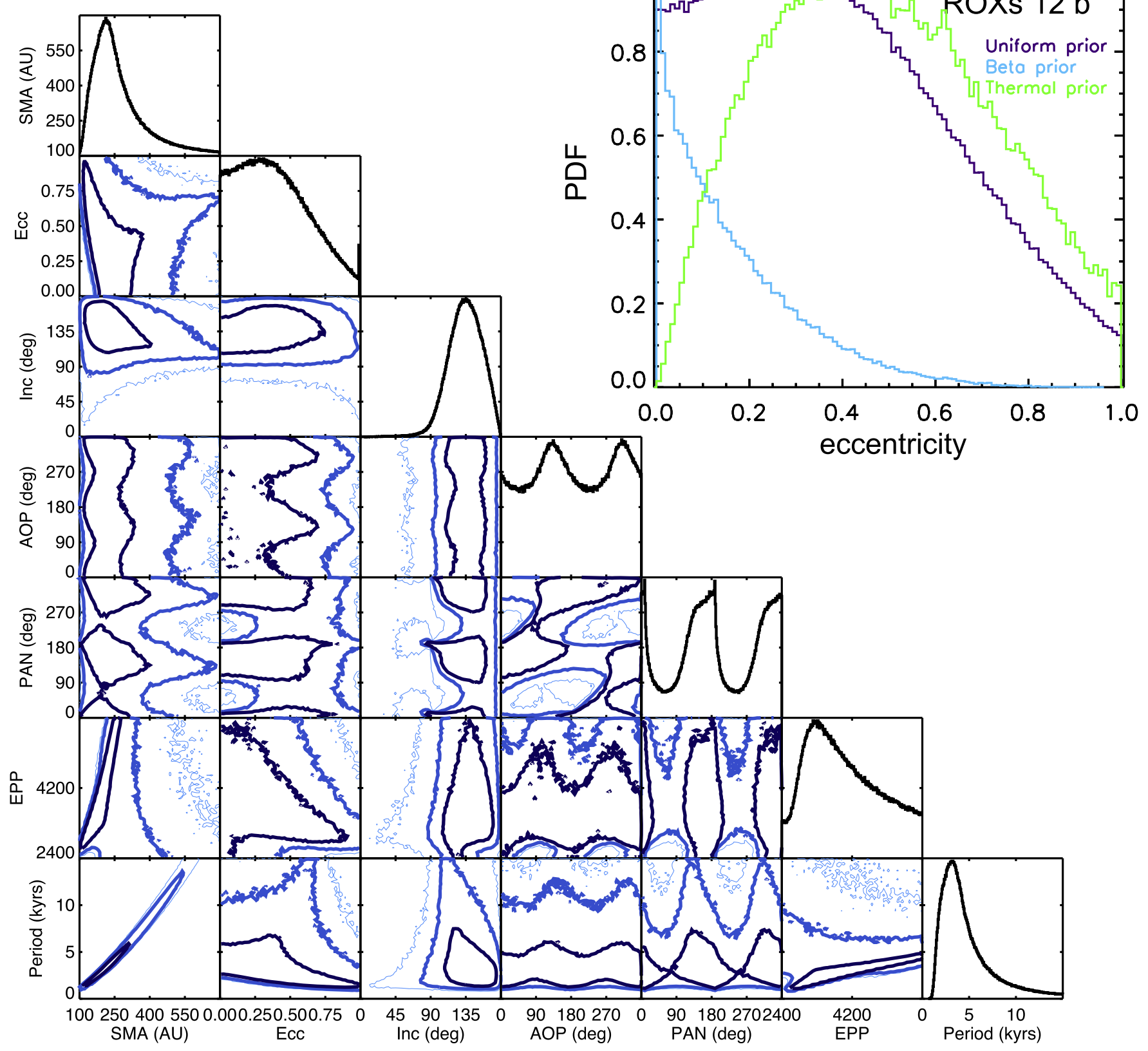

Figure 10. Marginalized one-dimensional posterior probability distributions of orbital parameters for ROXs $12 \mathrm{~b}$ along the diagonal, and two-dimensional covariances in off-diagonal elements. Parameters plotted are semi-major axis, eccentricity, inclination, argument of periastron, position angle of nodes, epoch of periastron passage, and period. In the covariance plots the dark to light blue contours denote locations with $68 \%, 95 \%$, and $99.7 \%$ of the probability enclosed. The most likely orbits have a semi-major axis of $\sim 200 \mathrm{au}, \sim 3000$ year period, and generally circular $(e \lesssim 0.5)$ and face on $(i \lesssim 70$ or $i \gtrsim 110)$. In the inset on the upper right, three different eccentricity posteriors are plotted corresponding to three different priors. The purple, light blue, and dark blue posteriors correspond to a uniform, thermal, and $\beta$ distribution respectively.

that end up outside of $100 \mathrm{au}$, their eccentricities are significantly pumped up to $>0.5$ (Scharf \& Menou 2009; Nagasawa \& Ida 2011). The fact that the eccentricity distributions for ROXs $42 \mathrm{~B}$ b and ROXs $12 \mathrm{~b}$ favor moderate to low eccentricities argues against the scattering hypothesis for these companions. Note that while a uniform prior on the eccentricity is used in these fits, the eccentricity posterior is significantly different. We can conclude that the eccentricity posterior is a reflection of the underlying companion eccentricity and not of the prior chosen. To further test this, we ran these orbit fits with two additional eccentricity priors, the $\beta$ distribution
(Kipping 2013) and the thermal distribution (Ambartsumian 1937). The thermal distribution of eccentricities, which is proportional to $2 e \mathrm{~d} e$, is the distribution that binary companions should follow if they are distributed solely as a function of energy. The eccentricity posteriors using these priors are overplotted with the eccentricity posterior found using a uniform prior in the top right plot in Figures 10 and 11. For both ROXs $12 \mathrm{~b}$ and ROXs 42B b, while the eccentricity posterior using the thermal distribution prior pushes to higher eccentricities, in general lower to moderate eccentricities are favored. 


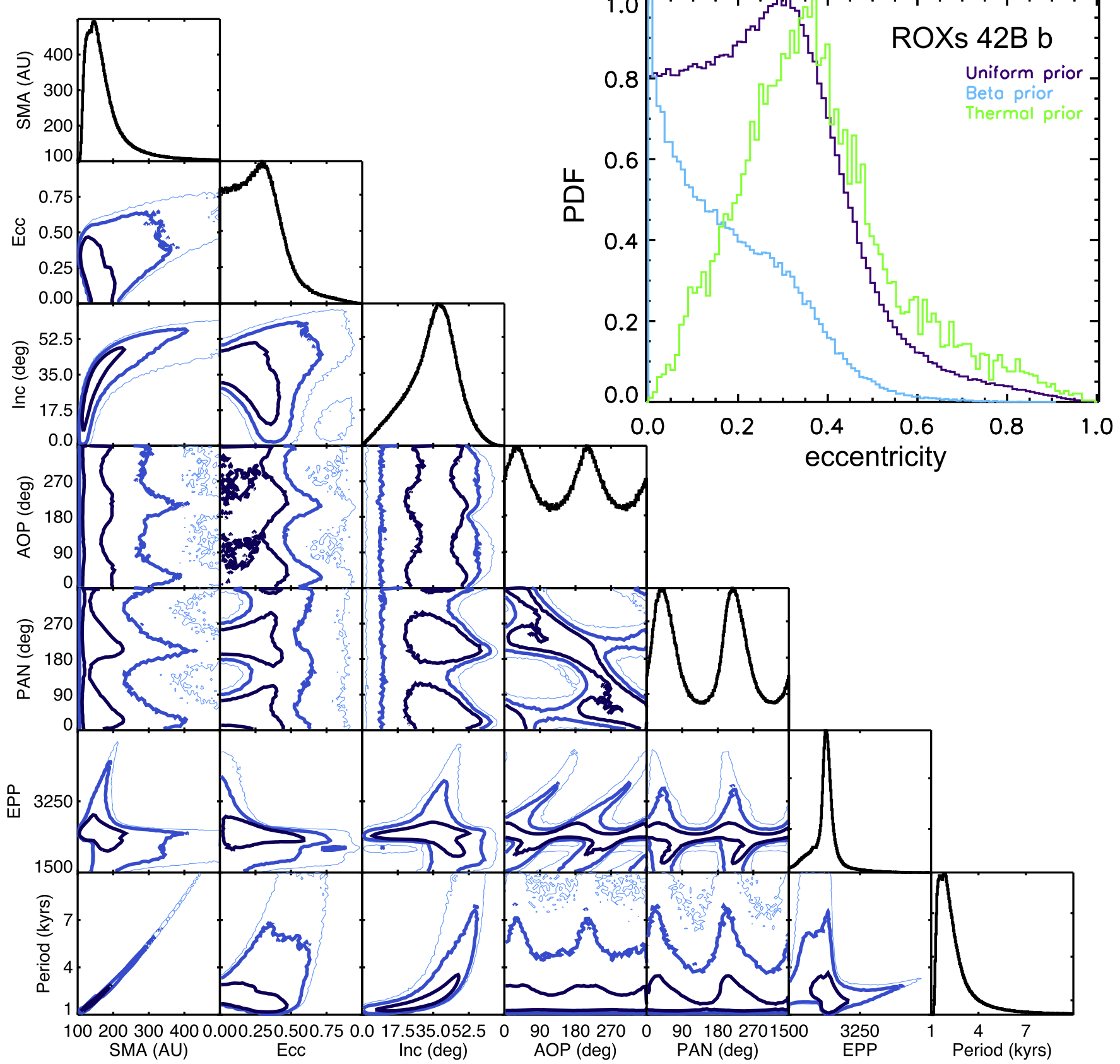

Figure 11. Orbital parameter posterior distributions for ROXs 42B b. The distributions peak for $\sim 150$ au, $\sim 2000$ year orbits. More circular orbits are preferred, with higher inclinations corresponding to longer periods. As in Figure 10, in the inset on the upper right, three different eccentricity posteriors are plotted corresponding to three different priors. The purple, light blue, and dark blue posteriors correspond to a uniform, thermal, and $\beta$ distribution respectively.

\subsection{Detection Probability}

We calculate the detection probability for additional companions in these eight systems over a range of masses and separations. Our contrast curves can be converted into sensitivity maps in mass and semi-major axis using evolutionary models, the age and distance of the system and the uncertainties on these values, and an underlying distribution of planet eccentricities. Following Bowler et al. (2015a), we generate a population of artificial companions on random, circular Keplerian orbits with a given mass and semi-major axis. Each synthetic planet is assigned an apparent magnitude using an interpolated grid of the Cond hot-start evolutionary models (Baraffe et al. 2003), the distance and age of the host star, and the companion mass. We use the Cond evolutionary models because they extend down to planetary masses, although we note that different models can vary significantly in their predictions for the same planet mass. We do not explicitly account for this model-dependent error in our final analysis. The fraction of companions falling above a contrast curve compared to those falling below it yields the fractional sensitivity at that grid point. We further take into account the fractional field of view coverage for each target, which is uniformly complete out to $4^{\prime \prime}$ for our sample and drops to zero beyond that. Iterating over masses between $0.5-100 M_{\text {Jup }}$ and semi-major axes between 1 and 1000 au yields sensitivity maps for each target, which are shown in Figure 12 for this sample. Depending on the distance and age of the target, our 

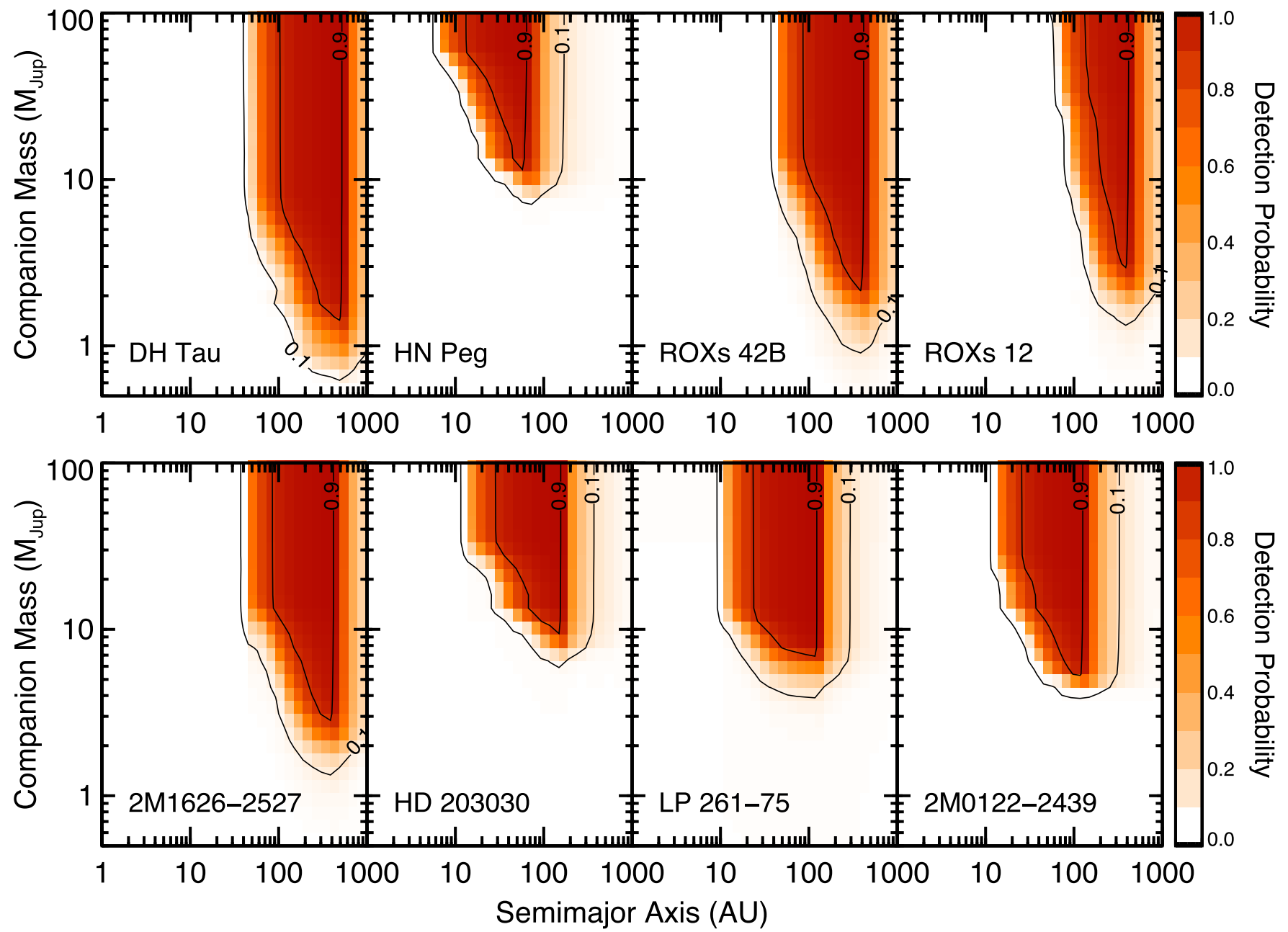

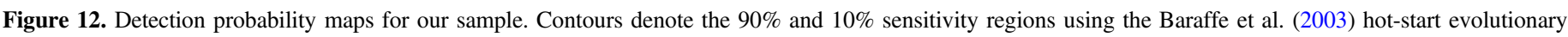

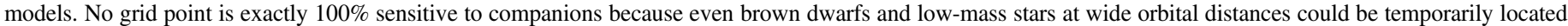

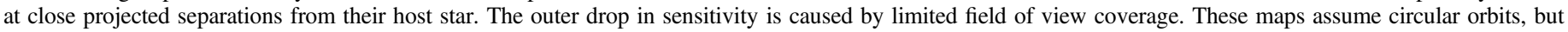
adopting modest eccentricities does not qualitatively change these results.

observations are generally sensitive to $1-10 M_{\text {Jup }}$ companions beyond about $30 \mathrm{au}$.

\section{DISCUSSION}

\subsection{Can Dynamical Scattering Explain This Population?}

Except for DH Tau cc1, which remains ambiguous, none of the new candidate companions detected in our sample are bound. While we can generally rule out the existence of massive scatterers above our detection limits (outside of 15-50 au for massive planets), we cannot unambiguously rule out scattering as a formation mechanism based on our results alone, since the massive scatterers might be located closer in. However, our results combined with complementary lines of evidence suggest that formation close to the host star plus subsequent scattering is probably not the dominant formation mechanism for these wide-separation PMCs. Note that in order for the scattering scenario to operate, there must be a body that is often at least as massive as these already massive PMCs closer in to the host stars (Veras \& Armitage 2004). We present a comprehensive list of this evidence below.

1. In this study, we do not find any potential scatterers down to $\sim 15-50$ au in this sample of seven systems which host wide-separation PMCs. Furthermore, other studies with comparably deep imaging of wide separation PMCs also did not find any potential scatterers in HD 106906 and 1 RXS 1609-2105 (e.g., Lafreniere et al. 2010; Bailey et al. 2014; Kalas et al. 2015; Lagrange et al. 2015). Efforts using non-redundant aperture masking techniques have probed higher masses $\left(>15 M_{\text {Jup }}\right)$ down to smaller separations $(>\sim 5 \mathrm{au})$, and have likewise found a dearth of inner companions (Kraus et al. 2011; Cheetham et al. 2015). If additional inner gas giant planets or brown dwarfs are present, they must be located within a few tens of au of their host stars.

2. Moderate to low eccentricities are favored for ROXs $12 \mathrm{~b}$ and ROXs 42B b, which both exhibit orbital motion. This is in contrast to the predictions of scattering simulations, which show that giant planets that get scattered out to $>100$ au typically have high eccentricities $>0.5$ (Scharf \& Menou 2009; Nagasawa \& Ida 2011).

3. From RV studies, it is clear that high-mass planets are rare. This is evident from the significantly negative power law in mass found by Cumming et al. (2008) for a sample of giant planets $0.3-10 M_{\text {Jup }}$ out to $3 \mathrm{au}$, where for a power law $m^{\alpha}, \alpha=-0.31 \pm 0.2$. Within this semi- 
major axis range, this power law implies that the occurrence rate of giant planets in the range 5-10 $M_{\text {Jup }}$ is $1.3 \%$. Similarly, work by Bryan et al. (2016) suggests that for a sample of gas giant planets outside $5 \mathrm{au}$, lowermass planets are more frequent than higher-mass planets. This implies that planets massive enough to be potential scatterers for the wide-separation directly imaged planets in this study $\left(>5 M_{\mathrm{Jup}}\right)$ are intrinsically rare in $\mathrm{RV}$ surveys. However, even if massive planets are disfavored generally, this doesn't necessarily mean that there would only be one super-massive planet per system. For example, perhaps unusual disk properties are required to form a $>5 M_{\text {Jup }}$ planet, but once this kind of disk is formed it is easy to form multiple massive planets at a range of separations. Given the low estimated occurrence rate of wide-separation PMCs (less than a few percent), this might be consistent with the low occurrence rate of massive planets found in RV surveys.

4. Dynamical interactions between planets preferentially scatter out lower-mass planets (Veras et al. 2009). However, lower-mass planets $\left(<5 M_{\mathrm{Jup}}\right)$ have not been discovered at distances greater than 100 au despite the fact that many surveys were sensitive down to a few Jupiter masses (e.g., Biller et al. 2013; Bowler et al. 2013). This implies that the companion mass function truncates at $\sim 5 M_{\text {Jup }}$, inconsistent with scattering.

5. Rough estimates of the occurrence rate of these massive ( $>5 M_{\text {Jup }}$ ) wide-separation PMCs yield at most a frequency of a few percent (Ireland et al. 2011; Aller et al. 2013). In contrast, scattering simulations, which began with 100 systems populated with 10 planets each with masses between 0.1 and $10 M_{\text {Jup }}$ drawn from a uniform distribution in $\log M$ and with separations $<30$ au drawn from a uniform distribution in semi-major axis, find that the occurrence rate of scattered planets from 1 to $10 M_{\text {Jup }}$ outside of 300 au is $\sim 0.2 \%$ at $\sim 10 \mathrm{Myr}$ (Scharf \& Menou 2009). Furthermore, of the $0.2 \%$ occurrence rate of all planets from 1 to $10 M_{\mathrm{Jup}}$ that get scattered beyond 300 au, only a few percent of that population are $>4 M_{\text {Jup }}$ (Veras et al. 2009). This implies that the occurrence rate of massive $\left(>5 M_{\text {Jup }}\right)$ scattered planets predicted by these simulations is of the order several hundredths of a percent, which is orders of magnitude smaller than any of the occurrence rate measurements from surveys thus far.

6. Many of these wide-separation PMCs are actively accreting from a circumplanetary disk (Bowler et al. 2011; Zhou et al. 2014). However, if these objects were dynamically scattered, we might expect the circumplanetary disk to be partially or completely stripped away (Bowler et al. 2011). This implies that many if not all of the PMCs that we find did not undergo such a violent evolution and were thus able to keep their disks.

Taken together, these lines of evidence indicate that the most likely origin for these wide-separation PMCs is in situ formation. Evidence for in situ formation, by cloud fragmentation or disk instability, includes the fact that PMCs have been found orbiting low-mass brown dwarfs with decidedly nonplanetary mass ratios, implying that the tail of the initial mass function appears to continue down to at least 5-10 $M_{\text {Jup }}$. In addition, Brandt et al. (2014) found that a single power law distribution is consistent with a sample of 5-70 $M_{\text {Jup }}$ objects from the SEEDS survey. Given that results from many other surveys are well fit by this same power law distribution, this suggests that this population immediately below the deuteriumburning threshold are the end of a smooth mass function, sharing a common origin with more massive brown dwarfs.

\subsection{Occurrence Rate}

We now consider the multiplicity of directly imaged planetary systems. Although we did not find any new companions in our sample, we can place an upper limit on the occurrence rate of inner, massive planets in systems with previously known wide-separation PMCs. Since we have no detections, our occurrence rate is simply:

$$
O=\frac{N_{m}}{N_{\mathrm{sys}}}
$$

where $N_{m}$ is the number of planets that we missed in our survey due to incompleteness, and $N_{\text {sys }}$ is the number of systems in our sample.

The number of planets that we missed due to survey incompleteness can be expressed as:

$$
\begin{aligned}
& N_{m} \\
& =\Sigma_{i=0}^{N_{\text {sys }}}\left[\int_{a_{1}}^{a_{2}} d \log a \int_{m_{1}}^{m_{2}} d \log m f(m, a)\left(1-P_{i}(m, a)\right)\right] .
\end{aligned}
$$

Here, $P_{i}(m, a)$ is the probability of detecting a planet of mass $m$ at semi-major axis $a$ for system $i$. We have these values for a grid of masses and semi-major axes from our detection probability calculations, described in Section 3.4 and shown in Figure 8. The quantity $f(m, a)$ is the assumed distribution in mass and semi-major axis for the population of planets whose occurrence rate we wish to calculate. In our calculation we adopt the underlying distribution in Clanton \& Gaudi (2015), which combines five different exoplanet surveys compiled using three different detection methods to derive a double power law distribution in mass and semi-major axis for giant planets. This power law takes the form:

$$
f(m, a)=\frac{d N}{d \log m_{p} d \log a}=A\left(\frac{m_{p}}{M_{\text {Sat }}}\right)^{\alpha}\left(\frac{a}{2.5 \mathrm{au}}\right)^{\beta} .
$$

In this equation, $A=0.21_{-0.15}^{+0.20}, \quad \alpha=-0.86_{-0.19}^{+0.21}$, and $\beta=1.1_{-1.4}^{+1.9}$. We note that this power law was derived specifically for M dwarf host stars. Out of our seven systems with previously confirmed PMCs, five of the host stars are M stars. We then create a $30 \times 30$ grid evenly spaced in logarithmic bins with masses from 1 to $100 M_{\text {Jup }}$ and semimajor axes ranging from 1 to $1000 \mathrm{au}$, and determine the power law distribution values at each grid point.

Since we want to determine the probability of finding an inner planet given that an outer PMC has been detected, we calculated the occurrence rate of PMCs between 5-15 $M_{\mathrm{Jup}}$ and from $40 \mathrm{au}$ to the location of each PMC. The inner limit on the separation was chosen because we are reasonably complete for massive planets beyond 40 au for most of our systems. In order to take into account the large uncertainties on the power law parameters, we calculated the occurrence rate using a Monte Carlo method with $10^{6}$ trials, each time drawing a new $A, \alpha$, 
and $\beta$ value from a Gaussian distribution with widths equal to the parameter uncertainties. This yielded a distribution of missed planets, which we converted to a distribution in occurrence rate.

We found that the $95 \%$ confidence upper limit on the occurrence rate of planetary companions interior to our sample of previously known wide separation PMCs is 54\%. This result assumes the companion distribution shown in Equation (8) as well as hot start evolutionary models. This first estimate of the occurrence rate upper limit will be better constrained with the discovery and analysis of more PMC systems. Note that covariances between parameters have not been taken into account in this method, which inflates our upper limit.

\section{CONCLUSIONS}

We conducted a deep ADI survey with NIRC2 at Keck in search of close-in substellar companions to a sample of seven systems with confirmed PMCs on extremely wide orbits (>100 au). We explored the possibility that the wide-separation PMCs formed closer in to their host stars and were subsequently scattered out to their present day locations by a more massive body in the system. In this survey we obtained deep imaging for each target, for the first time probing significantly lower masses and smaller separations in all systems.

Within our sample we found eight candidate companions. Using second epoch data, we measured the astrometry for each candidate and determined whether or not they were co-moving by using an MCMC technique that calculates robust uncertainties from the posterior distributions, without the systematics that occur when the reduced images are used.

Seven candidate companions are unequivocally background objects, while the candidate companion near DH Tau remains ambiguous. Although our results alone do not conclusively rule out formation closer in to the host star followed by scattering as a formation mechanism for these wide-separation PMCs, the totality of evidence suggests that scattering is not a dominant formation mechanism. Instead, formation of these objects in situ appears to be more likely.

If we wish to better understand how these wide separation PMCs formed, there are several possible approaches to consider. Gaia will allow us to carve out the immediate environment around these young stars, which has been extremely difficult with our current imaging capabilities (due to unfavorable contrasts close to the star), and radial velocity capabilities (due to high jitter values for young stars). Furthermore, studying the composition of these PMCs by obtaining high resolution spectra might allow us to distinguish among formation mechanisms (Konopacky et al. 2013; Barman et al. 2015). While the core accretion model predicts that planets should have enhanced metallicities relative to their host stars, formation via disk instability or molecular cloud fragmentation should result in compositions matching those of the host star. Finally, large high-contrast imaging surveys of young star forming regions conducted homogeneously would give us a more precise measurement of the occurrence rates and orbital architectures of this population of planetary-mass objects.

The data presented herein were obtained at the W.M. Keck Observatory, which is operated as a scientific partnership among the California Institute of Technology, the University of
California and the National Aeronautics and Space Administration. The Observatory was made possible by the generous financial support of the W.M. Keck Foundation. We acknowledge the efforts of the Keck Observatory staff. The authors wish to recognize and acknowledge the very significant cultural role and reverence that the summit of Mauna Kea has always had within the indigenous Hawaiian community. We are most fortunate to have the opportunity to conduct observations from this mountain.

\section{REFERENCES}

Alibert, Y., Mordasini, C., Benz, W., et al. 2005, A\&A, 434, 343 Aller, K. M., Kraus, A. L., Liu, M. C., et al. 2013, ApJ, 773, 63 Ambartsumian, V. A. 1937, AZh, 14, 207

Bailey, V., Meshkat, T., Reiter, M., et al. 2014, ApJL, 780, 1

Baraffe, I., Chabrier, G., Barman, T. S., et al. 2003, A\&A, 402, 701

Barman, T. S., Konopacky, Q. M., Macintosh, B., et al. 2015, ApJ, 804, 1

Bate, M. R. 2009, MNRAS, 392, 590

Bate, M. R. 2012, MNRAS, 419, 3115

Bate, M. R., Bonnell, I. A., \& Bromm, V. 2002, MNRAS, 332, L65

Beust, H., Bonnefoy, M., Maire, A. L., et al. 2016, A\&A, 587, 89

Biller, B. A., Liu, M. C., Wahhaj, Z., et al. 2013, ApJ, 777, 160

Boss, A. P. 2006, ApJL, 637, L137

Bowler, B. P., Liu, M. C., Kraus, A. L., et al. 2011, ApJ, 743, 148

Bowler, B. P., Liu, M. C., Kraus, A. L., et al. 2014, ApJ, 784, 1

Bowler, B. P., Liu, M. C., Shkolnik, E. L., et al. 2013, ApJ, 774, 55

Bowler, B. P., Liu, M. C., Shkolnik, E. L., et al. 2015a, ApJS, 216, 7 Bowler, B. P., Shkolnik, E. L., Liu, M. C., et al. 2015b, ApJ, 806, 62 Brandt, T. D., McElwain, M. W., Turner, E. L., et al. 2014, ApJ, 794, 159 Bryan, M. L., Knutson, H. A., Howard, A. W., et al. 2016, ApJ, 821, 89 Carlsberg Meridian Catalogue 2011, 15

Chauvin, G., Lagrange, A. M., Lacombe, F., et al. 2004, A\&A, 425, 29 Chauvin, G., Lagrange, A. M., Zuckerman, B., et al. 2005, A\&A, 438, 29 Cheetham, A. C., Kraus, A. L., Ireland, M. J., et al. 2015, ApJ, 813, 83 Clanton, C., \& Gaudi, S. 2015, arXiv:150804434C

Cumming, A., Butler, R. P., Marcy, G. W., et al. 2008, PASP, 120, 531

Currie, T., Cloutier, R., Brittain, S., et al. 2015, ApJL, 814, 27

Currie, T., Daemgen, S., Debes, J., et al. 2014, ApJ, 780, 30

Cutri, R. M., Skrutskie, M. F., van Dyk, S., et al. 2013a, 2MASS All Sky Catalog, VizieR Online Data Catalog

Cutri, R. M., Wright, E. L., Conrow, T., et al. 2013b, AllWISE Data Release De Rosa, R. J., Nielsen, E. L., Blunt, S. C., et al. 2015, ApJ, 814, 3

Dodson-Robinson, S. E., Veras, D., Ford, E. B., et al. 2009, ApJ, 707, 79 Ghez, A. M., Salim, S., Weinberg, N. N., et al. 2008, ApJ, 689, 1044

Ginski, C., Schmidt, T. O. B., Mugrauer, M., et al. 2014, MNRAS, 444, 2280 Ireland, M. J., Kraus, A., Martinache, F., et al. 2011, ApJ, 726, 113 Itoh, Y., Hayashi, M., Tamura, M., et al. 2005, ApJ, 620, 984

Kalas, P., Graham, J. R., Fitzgerald, M. P., et al. 2013, ApJ, 775, 56 Kalas, P. G., Rajan, A., Wang, J. J., et al. 2015, ApJ, 814, 1

Kipping, D. M. 2013, MNRAS, 434, L51

Konopacky, Q. M., Barman, T. S., Macintosh, B. A., \& Marois, C. 2013, Sci, 229, 1398

Kraus, A., \& Ireland, M. J. 2012, ApJ, 745, 5

Kraus, A. L., Ireland, M. J., Cieza, L. A., et al. 2014, ApJ, 781, 20

Kraus, A. L., Ireland, M. J., Martinache, F., et al. 2011, ApJ, 731, 1

Lafreniere, D., Jayawardhana, R., \& van Kerkwijk, M. H. 2010, ApJ, 719,497

Lagrange, A. M., Langlois, M., Gratton, R., et al. 2015, arXiv: 1510.02511

Lambrechts, M., \& Johansen, A. 2012, A\&A, 544, 32

Lodato, G., Delgado-Donate, E., \& Clarke, C. J. 2005, MNRAS, 364, 91

Low, C., \& Lynden-Bell, D. 1976, MNRAS, 176, 367

Luhman, K. L., Patten, B. M., Marengo, M., et al. 2007, ApJ, 654, 570

Luhman, K. L., Wilson, J. C., Brandner, W., et al. 2006, ApJ, 649, 894

Marois, C., Machintosh, B., \& Veran, J. P. 2010, Proc. SPIE, 7736, 77361

Marois, C., Macintosh, B., Barman, T., et al. 2008, Sci, 322, 1348

Metchev, S. A., \& Hillenbrand, L. A. 2006, ApJ, 651, 1166

Nagasawa, M., \& Ida, S. 2011, ApJ, 742, 2

Pollack, J. B., Hubickyj, O., Bodenheimer, P., et al. 1996, Icar, 124, 62

Quanz, S. P., Amara, A., Meyer, M. R., et al. 2015, ApJ, 807, 64

Ratzka, T., Kohler, R., \& Leinert, C. 2005, A\&A, 437, 611

Reid, I. N., \& Walkowicz, L. M. 2006, PASP, 118, 671

Sallum, S., Follette, K. B., Eisner, J. A., et al. 2015, Natur, 527, 342

Scharf, C., \& Menou, K. 2009, ApJL, 693, L113 
Service, M., Lu, J. R., Campbell, R., et al. 2016, PASP, 128, 095004

Skiff, B. A. 2013, General Catalogue of Stellar Spectral Classifications, VizieR Online Data Catalog

Soummer, R., Pueyo, L., \& Larkin, J. 2012, ApJL, 755, 28

Strom, K. M., Strom, W. E., Edwards, S., et al. 1989, AJ, 97, 1451

Veras, D., \& Armitage, P. J. 2004, MNRAS, 347, 613

Veras, D., Crepp, J. R., \& Ford, E. B. 2009, ApJ, 696, 1600
Vorobyov, E. I. 2013, A\&A, 552, 129

White, R. J., \& Ghez, A. M. 2001, ApJ, 556, 265

Yelda, S., Lu, J. R., Ghez, A. M., et al. 2010, ApJ, 725, 331

Zacharias, N., FInch, C. T., Girard, T. M., et al. 2012, UCAC4

Zacharias, N., Monet, D. G., Levine, S. E., et al. 2005, NOMAD Catalog, VizieR Online Data Catalog

Zhou, Y., Herczeg, G. J., Kraus, A. L., et al. 2014, ApJL, 783, L17 